\title{
Gradient and vorticity banding
}

\author{
Jan K. G. Dhont • Wim J. Briels
}

Received: 13 September 2007 / Accepted: 31 October 2007 / Published online: 21 February 2008

(C) Springer-Verlag 2008

\begin{abstract}
Banded structures" of macroscopic dimensions can be induced by simple shear flow in many different types of soft matter systems. Depending on whether these bands extend along the gradient or vorticity direction, the banding transition is referred to as "gradient banding" or "vorticity banding," respectively. The main features of gradient banding can be understood on the basis of a relatively simple constitutive equation. This minimal model for gradient banding will be discussed in some detail, and its predictions are shown to explain many of the experimentally observed features. The minimal model assumes a decrease of the shear stress of the homogeneously sheared system with increasing shear rate within a certain shear-rate interval. The possible microscopic origin of the severe shear-thinning behaviour that is necessary for the resulting nonmonotonic flow curves is discussed for a few particular systems. Deviations between experimental observations and predictions by the minimal model are due to obvious simplifications within the scope of the minimal model. The most serious simplifications are the neglect of concentration dependence of the shear stress (or on other degrees of freedom) and of the elastic contributions to the stress, normal stresses, and the possibility of shear-induced phase transitions. The consequences of coupling of stress and concentration will be analyzed in some detail. In contrast to predictions of the minimal model, when coupling to concentration is important, a flow instability can occur that
\end{abstract}

J. K. G. Dhont $(\bowtie)$

Forschungszentrum Jülich, IFF/ Weiche Materie,

52425 Jülich, Germany

e-mail: j.k.g.dhont@fz-juelich.de

W. J. Briels

Computational Biophysics, University of Twente,

Postbus 217, 7500 AE Enschede, The Netherlands does not require strong shear thinning. Gradient banding is sometimes also observed in glassy- and gel-like systems, as well as in shear-thickening systems. Possible mechanisms that could be at the origin of gradient-band formation in such systems are discussed. Gradient banding can also occur in strongly entangled polymeric systems. Banding in these systems is discussed on the basis of computer simulations. Vorticity banding is less well understood and less extensively investigated experimentally as compared to gradient banding. Possible scenarios that are at the origin of vorticity banding will be discussed. Among other systems, the observed vorticity-banding transition in rod-like colloids is discussed in some detail. It is argued, on the basis of experimental observations for these colloidal systems, that the vorticity-banding instability for such colloidal suspensions is probably related to an elastic instability, reminiscent of the Weissenberg effect in polymeric systems. This mechanism might explain vorticity banding in discontinuously shear-thickening systems and could be at work in other vorticity-banding systems as well. This overview does not include time-dependent phenomena like oscillations and chaotic behaviour.

Keywords Gradient banding - Vorticity banding . Shear banding

\section{Introduction}

Two types of banding instabilities may be distinguished: gradient banding and vorticity banding. In case of gradient banding in a Couette cell, the stationary state is one where two bands coexist, which extend in the gradient direction. A top view of such a banded state is schematically depicted in Fig. 1, left figure. The shear rate within each of the two 

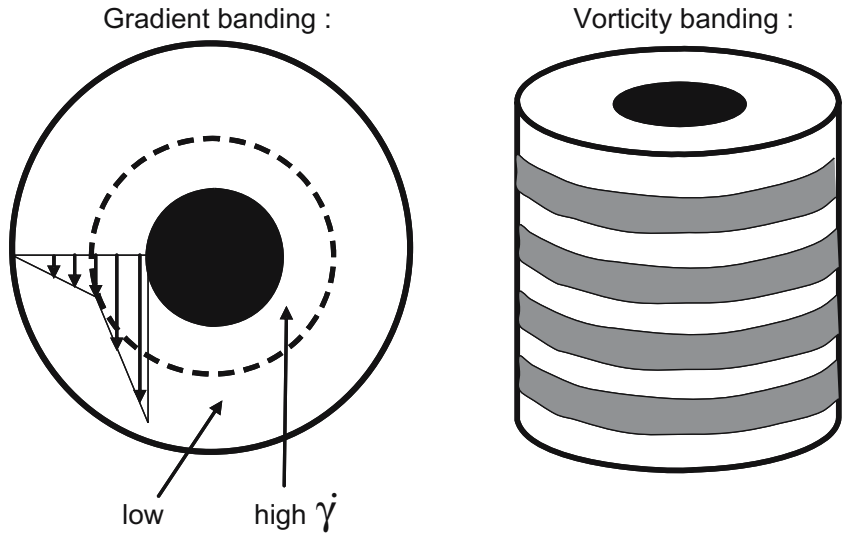

Fig. 1 Schematic of the morphology of the patterns formed in gradient banding (left figure), where the inner cylinder is supposed to rotate, and vorticity banding (right figure) in a Couette geometry

bands is essentially spatially constant, and the two bands are connected by a sharp "interface." In case of vorticity banding, regular bands are formed that are stacked in the vorticity direction, as depicted in Fig. 1, right figure. Depending on the system, these bands are visible because the two types of bands have a different turbidity or because they are both birefringent but with somewhat different orientations of their optical axes.

This overview consists of two main sections, one on gradient banding and one on vorticity banding.

In the section "Gradient banding," a quite simple, minimal model is discussed that already explains many of the experimental findings. Not included in the minimal model is a possible strong coupling of the stress to variations in concentration (or other degrees of freedom, like orientational order), elastic stresses, and normal stresses. The gradient-banding transition occurs when the shear stress (the flow-gradient component of the stress tensor) of the homogeneously sheared system (before banding occurs) decreases with increasing shear rate. The initial stages of band formation is predicted to proceed through a scenario that is similar to the Cahn-Hilliard gasliquid phase separation kinetics for spinodal decomposition. The omission of elastic stresses within the minimal model, however, is not allowed when band formation occurs on a time scale that is smaller than typical elastic relaxation times. Stress selection under controlled shear rate conditions can be quantified, and the minimal model explains why under controlled stress conditions no banding occurs. The necessary severe shear-thinning behaviour that complies with a decreasing stress with increasing shear rate can be connected to the very slow dynamics of degrees of freedom that strongly couple to the shear stress. A mechanism for severe shear thinning will be illustrated for rod-like colloidal particles that exhibit an isotropic-nematic phase transition. For worm-like micellar solutions, the flow behaviour of entanglement points and the resulting cou- pling of the length of the worms to shear flow can strongly enhance shear thinning as compared to colloidal rods. The response of entanglements in worm-like micellar systems to flow and the mechanism for the increase of the worm length with increasing shear rate are discussed on the basis of computer simulations. A strong dependence of the shear viscosity on concentration, which is not accounted for in the minimal model, gives the system an additional degree of freedom to shear band also under controlled stress conditions. This leads to different concentrations in the two bands. Gradient banding due to polymeric entanglements will be discussed on the basis of computer simulations on "resin particles," that is, particles with a small core that is covered by long polymeric chains. In addition to banding under controlled stress conditions, coupling to concentration can also lead to an instability without strong shearthinning behaviour. This type of instability could explain the experimentally observed gradient banding in some of the systems that do not exhibit strong shear thinning. Discontinuous shear thickening, when at a critical shear rate the stress jumps to a relatively high value as a result of the occurrence of a shear-induced viscous phase, can also give rise to gradient banding.

Much less is known about the origin of vorticity banding. The vorticity banding transition is, at least for some systems, possibly related to the development of normal stresses toward the inner cylinder of the Couette cell. As will be discussed, the mechanism is similar to that for the Weissenberg effect in polymer systems, where the role played by polymer chains in the Weissenberg effect is now played by inhomogeneities. The accompanying secondary rolling flow within the bands is indeed observed experimentally for rod-like colloids. The inhomogeneities can form through flocculation, early stage phase separation or through the nucleation of a shear-induced new phase. This might explain why discontinuous shearthickening systems, where a shear-induced viscous phase is formed beyond a critical shear rate, sometimes exhibit vorticity banding. Normal stresses along the gradient direction that lead to rolling flow might also originate from the interface in a gradient-banded state. In that case, first-gradient banding occurs, after which vorticity bands are formed. In case the shear rate is assumed to be a constant throughout the system, independent of position, a necessary condition for the stability of a vorticity-banded state is that the flow curve is multi-valued. The homogeneously sheared system should now be able to sustain different stresses (within the two types of bands) at the same shear rate. It is still unclear whether vorticity banding is generically due to an elastic type of instability (in which case, the bands are in internal rolling flow) or whether mass transport or reorganization of microstructural order plays an important role in some systems (which might comply with an essentially constant shear rate throughout the shear cell). 
Recent review papers that address banding phenomena include Olmsted (1999a), which is a comprehensive overview of the existing literature; Vermant (2003), where shearinduced bundles and strings, as well as near-critical and weakly aggregated colloids are discussed; and Cates and Fielding (2006) and Berret (2005), which extensively discuss the rheology and banding of micellar systems. Cates and Fielding (2006) also contains a section on time-dependent phenomena. A recent treatment of time-dependent phenomena can be found in Fielding (2007a).

\section{Gradient banding}

The most intensely studied systems that exhibit gradient banding are worm-like micellar systems (experiments on worm-like micelles can be found in Berret et al. 1997, 1998; Britton and Callaghan 1999; Fischer and Callaghan 2001; Britton et al. 1999; Salmon et al. 2003a; Herle et al. 2005; $\mathrm{Hu}$ and Lips 2005; Liberato et al. 2006; Yesilata et al. 2006; Miller and Rothstein 2007; and Manneville et al. 2007 and theoretical models for worms concerned with banding in Spenley et al. 1993 and 1996, and Vasquez et al. 2007). In some (diluted and concentrated) worm-like systems, wall slip is reported (Manneville et al. 2007; Salmon et al. 2003b). Gradient banding is also reported to occur in entangled polymers (Callaghan and Gil 2000; Britton and Callaghan 1997a; Tapadia et al. 2006), micellar cubic phases (Eiser et al. 2000a, b), supra-molecular polymer solutions (van der Gucht et al. 2006), transient networks (Michel et al. 2001), and thermotropic side chain liquid crystal polymers (Pujolle-Robic et al. 2002). Experiments indicate that gradient banding can also occur in hexagonal phases of surfactant solutions (Ramos et al. 2000). Gradient banding where a shear-induced second phase is involved is observed in lamellar surfactant systems, where an "onion phase" is induced (Bonn et al. 1998; Salmon et al. 2003c, d) in a semiflexible thermotropic liquid crystalline polymer (Mather et al. 1997), where a nematic phase is induced by flow, and in poly-crystalline colloids (Chen et al. 1992; Imhof et al. 1994; von Hünerbein et al. 1996; Palberg and Würth 1996; Preis et al. 1998), where crystals are shear melted beyond some critical shear rate. For the onion system, it is found that the location of the interface strongly fluctuates; the mechanism for which is still unclear (Salmon et al. 2003d). Interface fluctuations and periodic stress and rate response have been observed to accompany gradient banding in some cases (see, for example, Britton et al. 1999; Salmon et al. 2003a, d; Herle et al. 2005; Callaghan and Gil 2000; Manneville et al. 2004a; $\mathrm{Hu}$ et al. 2002; Rofe et al. 1996; Lerouge et al. 2006). It is still an open question to what extent dynamical slip-stick phenomena play a role in the observed interface fluctuations. Such time-dependent phe- nomena will not be addressed in the present overview (see Fielding 2007a for an overview of time-dependent shearbanding phenomena).

Spatially resolved velocity profiles that are inhomogeneous along the gradient direction can be measured with nuclear magnetic resonance (NMR; Britton et al. 1999; Callaghan and Gil 2000; Britton and Callaghan 1997b; Callaghan 1999), heterodyne dynamic light scattering, and particle tracking velocimetry (Salmon et al. 2003a, e; Hu and Lips 2005; Miller and Rothstein 2007), and ultrasound (Manneville et al. 2004a, b), whereas structural changes can be inferred from birefringence/dichroism measurements (Berret et al. 1997; Hu and Lips 2005; Liberato et al. 2006; Miller and Rothstein 2007; Fischer and Callaghan 2000; Lerouge et al. 1998, 2000; Decruppe et al. 1997, 2001; Cappelaere et al. 1997), light transmission (Lerouge et al. 2000), and microscopy (Herle et al. 2005; Hu and Lips 2005; Imhof et al. 1994). Light and neutron scattering have been used to probe the (superposition of) microstructural features of shear bands (Herle et al. 2005; Liberato et al. 2006; Decruppe et al. 2001; Cappelaere et al. 1997).

Banded flow profiles are ofcourse different in different geometries. The velocity profiles in a polymer solution (semi-dilute polyacrylamide solutions) in a cone-plate geometry, as observed by NMR (Britton and Callaghan 1997a), and of poly-crystalline charged colloids in a pipe geometry (Palberg and Würth 1996; Preis et al. 1998) are more complicated than in a small-gap Couette cell. For large cone angles in a cone-plate geometry, the free fluidair interface leads to complicated flow profiles even for Newtonian fluids. In a wide-gap Couette cell, there is a considerable natural variation of the shear rate within the gap. The local shear rate, before banding occurred, may in some regions be inside and some regions outside the shearrate range where gradient banding occurs. That the observed flow profiles in Fischer and Callaghan (2001, 2000) do not exhibit the expected two-band structure is probably not due to the large size of the gap. Instead, these flow profiles have been explained (Fischer and Callaghan 2001) by postulating that, at high shear rates, a viscous phase is induced by the flow, which subsequently relaxes to a less viscous state. It remains unclear what the nature of this transient, highly viscous state is (see, however, "The microscopic origin of strong shear thinning"). Alternatively, secondary flow can have a strong impact on velocity profiles in certain geometries (Kumar and Larson 2000). Apart from the time-dependent phenomena, many of the experimental observations can be understood on the basis of a quite simple model (Dhont 1999; Olmsted and Lu 1999a; Lu et al. 2000). This "minimal model" will be discussed in "A minimal model: stress selection and banding kinetics." The possible microscopic mechanisms that lead to strong shear thinning, which is required for 
gradient banding within the realm of the minimum model, will be discussed in "The microscopic origin of strong shear thinning." The experimentally observed deviations from the predictions of this minimal model can be attributed to the dependence of the shear viscosity on order parameters like concentration and orientational order. This "coupling to concentration" and its consequences for gradient banding is discussed in "Coupling to concentration." Special attention is given to shear-thickening systems in "Gradient banding of shear-thickening systems."

A minimal model: stress selection and banding kinetics

Here, we will present the simplest model (Dhont 1999; Olmsted and Lu 1999a; Lu et al. 2000) that exhibits many of the gradient-banding features that are seen experimentally. Consider a laminar flow in a two-plate geometry in the $x$ direction with its gradient in the $y$ direction. Neglecting variations of the flow velocity $u$ in the $x$ and $z$ directions, the Navier-Stokes equation simply reads:

$\rho_{m} \frac{\partial u(y, t)}{\partial t}=\frac{\partial \sum(y, t)}{\partial y}$,

where $\rho_{m}$ is the mass density of the fluid under consideration and $\Sigma$ is the shear stress. The standard expression for the stress is $\dot{\gamma} \eta$, with $\dot{\gamma}$ as the local shear rate and $\eta$ the shear viscosity. This expression for the shear stress is the leading term in a formal expansion of the stress with respect to spatial derivatives of the fluid flow velocity. For smooth variations of the velocity, this expression is sufficient to describe flow phenomena. That is, this would be the expression for the stress of a homogeneously sheared system before banding occurs. This standard shear stress will be denoted by $\sigma=\dot{\gamma} \eta$. Experiments indicate that this standard stress exhibits a van der Waals loop-like behaviour as depicted in Fig. 2 for systems that show gradient banding. It is important to note that this is the flow curve for the homogeneously sheared system before banding had time to occur. The standard expression for the stress cannot describe a stationary state where $d \sigma(y) / d y=0$, as the van der Waals loop is probed when crossing the interface that connects the two bands. Within the interface, spatial variations of the flow velocity fields are probably too large to restrict the dependence of the stress to first-order flowvelocity derivatives. It therefore seems natural to extend the formal expansion to include the next higher order gradient term. From symmetry considerations, it is readily seen that this expansion reads (Dhont 1999; Olmsted and Lu 1999a; Lu et al. 2000):

$\sum(y, t)=\eta(\dot{\gamma}(y, t)) \dot{\gamma}(y, t)-\kappa(\dot{\gamma}(y, t)) \frac{\partial^{2} \dot{\gamma}(y, t)}{\partial y^{2}}$.
The constant of proportionality $\kappa$ of the second-order derivative of the shear rate is referred to as the shearcurvature viscosity (Dhont 1999). Such shear-curvature contributions to the stress may arise implicitly from diffusion of stress elements, in which case an equation of motion for the stress tensor contains a diffusive term (Spenley et al. 1996; Lu et al. 2000; Radulescu et al. 2003; Yuan 1999; El-Kareh and Leal 1989), proportional to the so-called stress-diffusion coefficient. There is no simple connection between the shear-curvature viscosity and the stress-diffusion coefficient, as the stress-diffusion contribution is formulated in terms of the Laplace of the stress tensor instead of simply the Laplace of the shear rate as in Eq. 2. Assuming a banded velocity profile, it is easily verified (Dhont 1999) that a $y$-independent stress, also through the interface, is only possible if

$\kappa>0$.

This is why in Eq. 2, the higher order term is provided with a minus sign. Furthermore, the shear-curvature viscosity tends to 0 in a shear-rate range where the viscosity attains its high shear-rate value. This can be seen as follows. Suppose that local microstructural order is saturated by the large local shear rates. That is, applying an even larger local shear rate will not change microstructural order. The stress must then be independent of higher order derivatives of the flow velocity, as such contributions account for local gradients in microstructural order. Hence, even though $\partial^{2} \dot{\gamma} / \partial y^{2}$ may be large, the shear-curvature contribution cannot contribute to the shear stress in Eq. 2 for large shear rates, which implies that $\kappa=0$. Hence,

$\lim _{\dot{\gamma} \rightarrow \infty} \kappa(\dot{\gamma})=0$.

The shear-rate range in which $\kappa$ shear thins to zero is the same as where the shear viscosity reaches its high shear-rate limit.

There are four obvious omissions in the minimal model:

1. The concentration dependence of the shear stress is neglected. The consequences of such as concentration dependence will be discussed in "Coupling to concentration."

2. Elastic contributions to the stress are neglected. This leads to wrong predictions for banding kinetics when band formation occurs on a time scale that is smaller than the typical relaxation times of elastic stresses. Such contributions are considered in Goddard (2003) and will not be discussed here further.

3. Flow-induced phase transitions are not accounted for. The possible role played by such transitions in gradient-band formation is discussed in "Gradient banding of shear-thickening systems." 
4. The minimal model is a scalar theory that neglects normal stresses. Normal stresses may affect stress selection and can give rise to time-dependent phenomena, which will not be addressed here.

A stress selection rule can be extracted from the constitutive Eq. 2. Suppose that a stationary banded structure exists (as depicted in Fig. 1, left figure). Let $\Sigma_{\text {stat }}$ be the constant stress that is attained throughout the gap (in a two-plate geometry) in the stationary state,

$\sum_{\text {stat }}=\eta(\dot{\gamma}(y)) \dot{\gamma}(y)-\kappa(\dot{\gamma}(y)) \frac{d^{2} \dot{\gamma}(y)}{d y^{2}}$.

When, in the stationary state, the shear rate within the interior of the two bands is spatially constant, we have

$$
\int_{\dot{\gamma}_{-}}^{\dot{\gamma}_{+}} d \dot{\gamma} \frac{d^{2} \dot{\gamma}(y)}{d y^{2}}=\frac{1}{2} \int_{y_{-}}^{y_{+}} d y \frac{d}{d y}\left(\frac{d \dot{\gamma}(y)}{d y}\right)^{2}=0,
$$

provided that the shear rate is a monotonic function of position. Here, $\dot{\gamma}_{+}$and $\dot{\gamma}_{-}$are the shear rates in the high shear-rate and low shear-rate band, respectively, whereas $y_{+}$ and $y_{-}$are positions within the two corresponding bands. Integration of Eq. 5 thus leads to

$$
\int_{\dot{\gamma}_{-}}^{\dot{\gamma}_{+}} d \dot{\gamma} \frac{\eta(\dot{\gamma}) \dot{\gamma}-\sum_{\text {stat }}}{\kappa(\dot{\gamma})}=0,
$$

This is the stress-selection rule within the minimal model (Dhont 1999; Olmsted and Lu 1999a; Lu et al. 2000). The system will select a stress $\Sigma_{\text {stat }}$ in the stationary state such that it satisfies Eq. 7. Had the shear-curvature viscosity been independent of the shear rate, then this stress-selection rule would have implied an equal-area Maxwell construction. According to Eq. 4, however, the shear-curvature viscosity is shear-rate dependent, which shifts the selected stress to somewhat lower values as compared to the equal-area stress, as depicted in Fig. 2. The shear-curvature viscosity is only relevant within the interface, except for small-scale flow geometries with a size comparable to the interface width (an example of this will be discussed later in this section). The selected stress thus depends to some extent on interfacial properties. Hence, there is no quantity that depends only on the bulk properties of the two bands, like a "chemical potential," that sets the selected stress.

Suppose that the solid line in Fig. 3 is the stationary solution of the Navier-Stokes equation $(1,2)$ for the banded flow profile for one particular applied shear rate. A whole set of solutions, each corresponding to a given applied shear rate, can then be constructed by simply shifting the interface to the right for a lower applied shear rate or to the left for a higher shear rate (see, for example, the dotted line

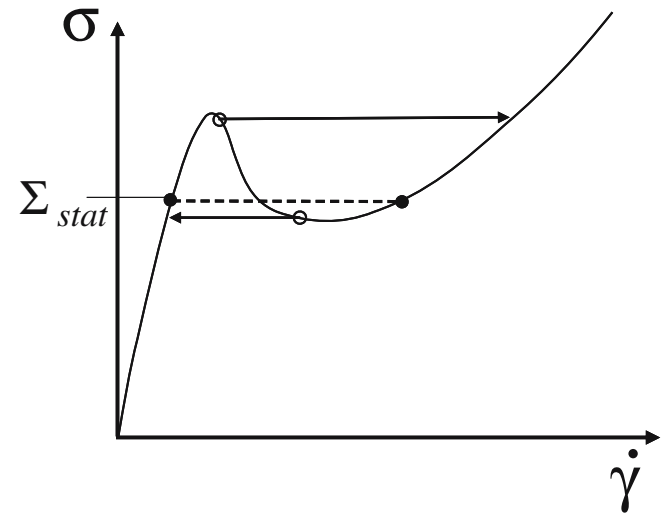

Fig. 2 The "van der Waals loop-like" behaviour of the standard shear stress $\sigma$, that is, the stress of the homogeneously sheared system, before banding occurred, as a function of the shear rate $\dot{\gamma}$. The dashed horizontal line marks the selected stress in the stationary state under controlled shear-rate conditions according to the "modified" equalarea Maxwell construction (7). The horizontal arrows correspond to bottom and top jumps that are observed under controlled-stress conditions when the imposed stress is not equal to the modified equal-area stress

in Fig. 3). Note that this implies that, within the present minimal model, the shear rates $\gamma_{ \pm}$within the two bands are independent of the applied shear rate. Together with Eq. 7, this explains the often observed stress plateau within the banding region. Note that it also follows from this consideration that the relative portions of the bands as a function of the applied shear rate obey a lever rule. Such a lever rule and/or the independence of the shear rates within the two bands on the applied shear rate is confirmed experimentally in, for example, Berret et al. (1997), Britton et al. (1999), Salmon et al. (2003a), Liberato et al. (2006), Cappelaere et al. (1997), Porte et al. (1997), and by computer simulations (van den Noort and Briels 2007).

The system has the freedom to select its stress when the shear rate is controlled during an experiment. When the stress is controlled and the applied stress is different from $\Sigma_{\text {stat }}$ as given in Eq. 7, no true shear banding can occur. When the shear rate is constant within the bands, the stress must be equal to $\Sigma_{\text {stat. }}$ Hence, shear banding cannot occur in a stress-controlled experiment. There are now two possible scenarios: (1) the flow profile always has a uniform shear rate, which, with increasing stress, jumps from its maximum to the stable branch at higher shear rate (and similarly for decreasing stress) or (2) a flow profile exists which is neither a banded state nor a state where the shear rate is homogeneous but rather a flow profile where the shear rate varies throughout the entire gap of the cell. "Bottom and top jumps" under controlled stress conditions are observed in, for example, micellar systems (Yesilata et al. 2006; Cappelaere et al. 1997; see also Fig. 3 in Lerouge et al. 2000), probably in a colloidal crystalline system 


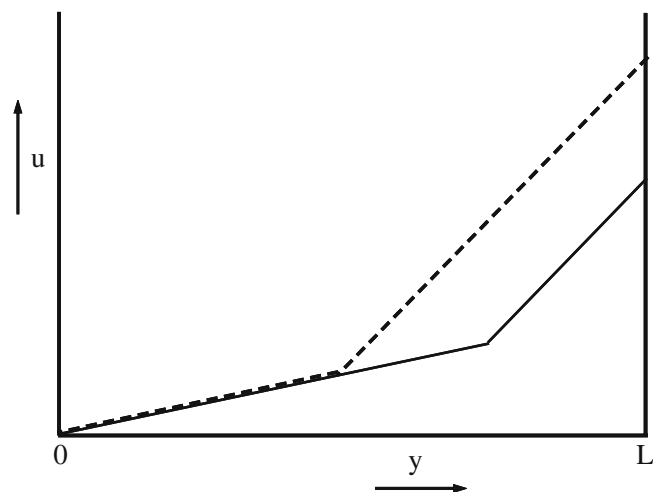

Fig. 3 A banded velocity profile that is obtained by a mere translation of the interface remains a solution of the Navier-Stokes equation (provided that coupling to a conserved quantity, like concentration, is irrelevant)

(Chen et al. 1992), in supra-molecular polymer solutions (van der Gucht et al. 2006), and in dispersions of clay particles (ten Brinke et al. 2007). The top jumps in Cappelaere et al. (1997), however, can also be due to a shear-induced phase transition. The jumps are indicated in Fig. 2 by the horizontal arrows. There are, however, experiments where banding is seen also under controlled stress conditions. As will be discussed in "Coupling to concentration," strong coupling of concentration to stress can lead to banded states also under controlled stress conditions.

The question now is under which conditions a linear flow profile will be unstable. To answer this question, we perform a linear stability analysis on the Navier-Stokes Eq. 1. Let $\dot{\gamma}_{0}$ denote the applied shear rate. The "natural" flow profile in a two-plate geometry would be one where the velocity $u$ is equal to $\dot{\gamma}_{0} y$, where $y$ is again the distance from the lower, stationary plate. Consider a flow profile where this natural velocity is perturbed, that is, $u(y, t)=\dot{\gamma}_{0} y+\delta u(y, t)$, where $\delta u(y, t)$ is the small perturbation. Substitution into Eqs. 1 and 2 and linearization with respect to $\delta u$, assuming stick-boundary conditions, readily leads to

$\delta u(y, t)=\sum_{n=1}^{\infty} \alpha_{n} \sin \left\{k_{n} y\right\} \exp \left\{-\Lambda_{n} k_{n}^{2} t\right\}$,

where the coefficients $a_{n}$ are determined by the initial form of the perturbation, the wave vectors $k_{n}=n \pi / L$ (with $L$ the gapwidth and $n=1,2, \ldots)$, and

$\Lambda_{n}=\frac{1}{\rho_{m}}\left[\frac{d \sigma\left(\dot{\gamma}_{0}\right)}{d \dot{\gamma_{0}}}+\kappa\left(\dot{\gamma}_{0}\right) k_{n}^{2}\right]$.
Clearly, the natural, linear flow profile is unstable when

$\frac{d \sigma\left(\dot{\gamma}_{0}\right)}{d \dot{\gamma}_{0}} \leq-\kappa\left(\dot{\gamma}_{0}\right) k_{n}^{2}$,

for $n=1$ (and possibly $n>1$ ). In case the right hand-side of Eq. 10 is very small, this complies with experimental findings that banding occurs when the slope of the standard stress $\sigma$ (which is the stress of the homogeneously sheared system) versus the applied shear rate is negative [this is found experimentally for worm-like micelles (Berret et al. 1997; Britton and Callaghan 1999; Fischer and Callaghan 2001; Britton et al. 1999; Salmon et al. 2003a), polycrystalline colloids (Imhof et al. 1994; von Hünerbein et al. 1996; Palberg and Würth 1996), micellar cubic crystals (Eiser et al. 2000a, b), and most probably this scenario also applies for semi-dilute polyacrylamide solutions (Callaghan and Gil 2000; Britton and Callaghan 1997a)]. The shearrate range where the homogeneously sheared system is unstable is marked by the two points $O$ in Fig. 2. According to Eqs. 3 and 10, these two points are not exactly located on the maximum and minimum of the van der Waals loop.

Note that, when the gap width $L$ is smaller than the "critical gap width" (with $\min _{y_{0}}$ referring to the minimum value for shear rates $\dot{\gamma}_{0}$ where $\left.d \sigma\left(\dot{\gamma}_{0}\right) / d \dot{\gamma}_{0}<0\right)$,

$L_{c r i t}=\min _{\dot{\gamma}_{0}} \sqrt{-\frac{\pi \kappa\left(\dot{\gamma}_{0}\right)}{d \sigma\left(\dot{\gamma}_{0}\right) / d \dot{\gamma}_{0}}}$,

so that the inequality in Eq. 10 is never satisfied for $n=1$, the natural, linear flow profile is always stable. In that case, "the smallest unstable wavelength does not fit into the gap," and one would be able to measure the entire van der Waals loop as sketched in Fig. 2 without having to worry about ongoing banding. For small values of the shear-curvature viscosity, the corresponding gap width will probably be too small for the realization of such a shear cell.

Numerical integration of the equation of motion (Eqs. 1 and 2) under controlled shear-rate conditions, where the applied shear rate $\dot{\gamma}_{0}$ is fixed, indeed leads to regions (the "bands") where the shear rate is constant, as shown in Fig. 4. The upper figure specifies two initial, small perturbations of the natural flow velocity. The right column of the figures shows the flow velocity $\Delta u=u-\dot{\gamma}_{0} y$ relative to the linear flow velocity for various times in terms of the dimensionless time $\tau=t \eta_{0} / \rho_{m} L^{2}$. The shearrate dependence of the standard stress and the shearcurvature viscosity used in this calculation are assumed to 


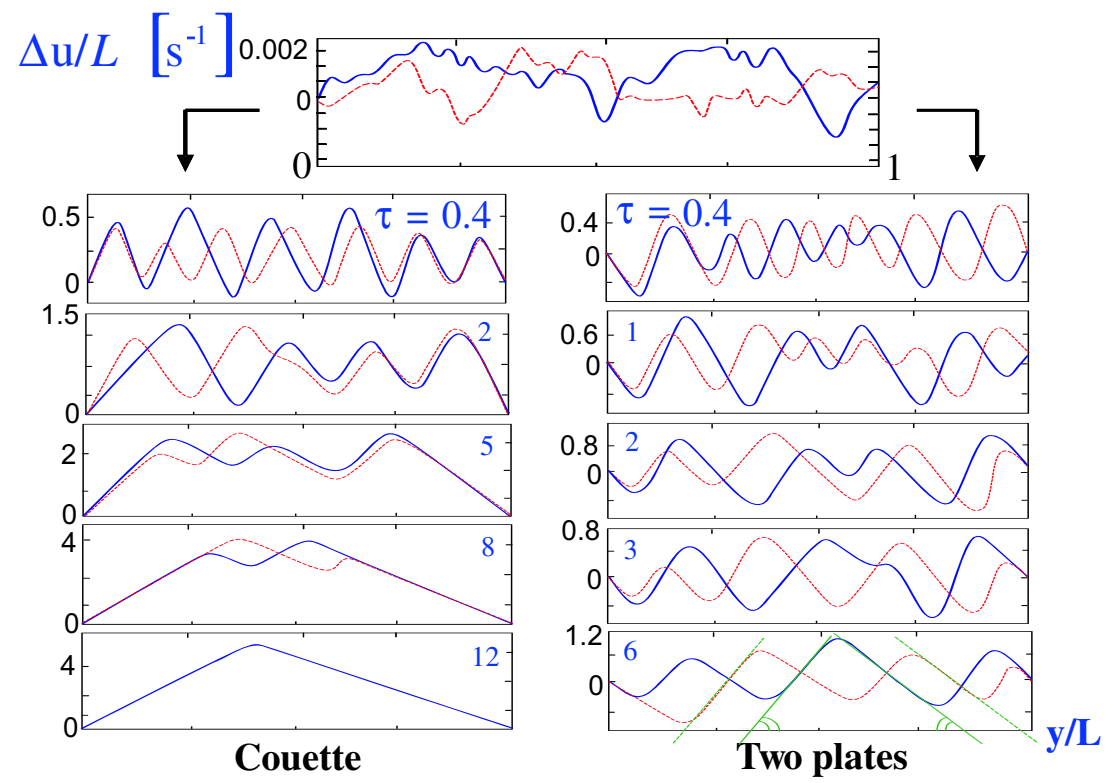

Fig. 4 The kinetics of gradient-band formation, within the scope of the minimal model, for a Couette geometry (left figure) and a two-parallel plate geometry (right figure). Plotted is $\Delta u / L=\left[u-\dot{\gamma}_{0} y\right] / L$, with $u$ the actual flow velocity, $\gamma_{0}$ the applied overall shear rate, $y$ the distance from the stationary outer cylinder and the stationary lower plate for the two geometries, respectively, and $L$ is the gap width. The inner cylinder is

follow the simplest possible forms that agree with conditions 3 and 4:

$\sigma(\dot{\gamma})=\dot{\gamma} \frac{\eta_{0}+\beta \eta_{\infty} \dot{\gamma}^{2}}{1+\beta \dot{\gamma}^{2}}, \kappa(\dot{\gamma})=\frac{\kappa_{0}}{1+\beta \dot{\gamma}^{2}}$,

with $\eta_{0}=20 \mathrm{~Pa} s$ as the zero-shear viscosity, $\eta_{\infty}=1 \mathrm{~Pa} s$ the infinite-shear viscosity, and $\beta=0.05 \mathrm{~s}^{2}$ is the parameter that sets the shear-thinning region. Furthermore, the zeroshear curvature viscosity $\kappa_{0} / L^{2}=1.710^{-3} \mathrm{~N} \mathrm{~s} \mathrm{~m}^{-2}$ is chosen such that the gap width is ten times larger than the critical gap width in Eq. 11. As can be seen from Fig. 4 (right column of plots), two different initial conditions lead to two different stationary-banded structures in case of a two-plate geometry. Clearly, for a two-plate geometry, any combination of bands and interfaces solves the NavierStokes equation. For a Couette geometry, however, the interfaces merge, as can be seen from the left column of Fig. 4. The ratio $a=L / R_{\mathrm{i}}$ of the gap width $L$ over the radius $R_{\mathrm{i}}$ of the inner cylinder here is chosen equal to $1 / 10$. The natural curvature of flow lines in a Couette geometry drives all the interfaces that are formed during the initial stages of banding together, leading to a well-defined, unique stationary banded state with just two bands and a single interface. The flow profiles in the two bands are the "natural profiles" for a Couette geometry, with a slight increase of the local shear rate toward the inner cylinder. Merging of the various interfaces is faster when the parameter $\alpha$ is larger, that is,

rotating anti-clockwise. The upper figure gives the two randomly generated initial flow profiles. The lower figures are velocity profiles for various times in terms of the dimensionless time $\tau=t \eta_{0} / \rho_{m} L^{2}$. For the Couette geometry, the ratio of the gap width to the radius of the inner cylinder is taken equal to $1 / 10$

when gradients in the local shear rate for the natural flow profile are larger.

During the initial stages of banding, it follows from Eqs. 8 and 9) that the growth rate $\Gamma_{n}$ of a sinusoidal velocity variation with a wavelength $\lambda_{n}=2 \pi / k_{n}$ is given by

$\Gamma_{n} \equiv-\Lambda_{n} k_{n}^{2}=-\frac{1}{\rho_{m}}\left[\frac{d \sigma\left(\dot{\gamma}_{0}\right)}{d \dot{\gamma}_{0}}+\kappa\left(\dot{\gamma}_{0}\right) k_{n}^{2}\right] k_{n}^{2}$.

The sinusoidal component of the velocity profile that grows fastest therefore corresponds to the wave vector:

$k_{n, \max }=\sqrt{-\frac{d \sigma\left(\dot{\gamma_{0}}\right) / d \dot{\gamma_{0}}}{2 \kappa\left(\dot{\gamma_{0}}\right)} .}$

As can be seen from Fig. 4, there is indeed a welldefined wavelength $\lambda_{n, \max }=2 \pi / k_{n, \max }$ that grows fastest, independent of the initial condition. Note that when it is possible to measure the fastest growing wavelength, this would be a means to measure the shear-curvature viscosity (provided that elastic stresses can be neglected during band formation). The shear-curvature contribution in Eq. 2 to the stress prevents very small wavelengths to grow in time. Velocity variations with a wave vector larger than the critical wave vector $k_{n, \text { crit }}=\sqrt{2} k_{n, \max }$ decay instead of grow. The initial growth scenario, within the simplifications 
involved in the minimal model, is formally very similar to the Cahn-Hilliard theory for gas-liquid demixing. The few experiments that report on the kinetics of gradient banding do not see this "Cahn-Hilliard" type of scenario (Hu and Lips 2005; Radulescu et al. 2003). The time interval over which the initial band-formation stage extends might be too short, elastic stresses could play an important role, and/or coupling to concentration may affect band-formation kinetics. Elasticity and coupling of stress to concentration can give rise to an initial maximum growth rate that is essentially constant within a considerable wave-vector range, so that there is not a single wave vector that grows most fast (Fielding and Olmsted 2003a, b). A non-Cahn-Hilliard-like demixing scenario is predicted for a Johnson-Segalman fluid, without the shear-curvature contribution, when the interface is treated as a point of discontinuity between the bands (Georgiou and Vlassopoulos 1998). Within the same Johnson-Segalman approach, Radulescu et al. (1999) and Olmsted et al. (2000) investigate the effects of a stress diffusion contribution on predicted stationary states.

A numerical value for the shear-curvature viscosity for a micellar system has been determined in Masselon et al. (2008) from stationary velocity profile measurements in a straight microchannel. The linear extent of the channel is so small that the higher order gradient contribution to the stress in Eq. 2 is important in relatively large regions within the channel. Velocity profiles could be accurately described with the constitutive relation (2) with a single, shear-rate independent value of $\kappa=2.110^{-10} N s$ for the shearcurvature viscosity. The shear-rate range probed by these experiments lies probably within the range where $\kappa$ is not yet shear thinning. Numerical values for the stress diffusion coefficient have been obtained experimentally for a wormlike micellar system in Radulescu et al. (2003) from the kinetics of band formation. In this case, it is probably essential to account for elastic contributions to the stress.

In analogy with the thermodynamic theory of phase separation (in the absence of flow), one can define gradient shear-banding spinodals and binodals. Spinodal-banding points can be defined as the shear rates where the natural flow profile becomes unstable. These are the points $\circ$ in Fig. 2, which can be calculated from Eq. 10. Binodal-banding points are the shear rates of the two coexisting bands. These are the points - in Fig. 2, which can be obtained from the modified equal area construction in Eq. 7. In the "meta-stable region," between the spinodal and binodal points, the natural flow profile can persist for some time. The true stationary state, however, is a banded state. The analog between thermodynamic and banding spinodals and binodals has been inferred experimentally in Berret and Porte (1999).

In case of (thermodynamic) phase separation from the meta-stable region, there is an energy barrier to be overcome by thermal fluctuations to create nuclei that initiate phase separation. In the present case of gradient banding, it is not clear whether such an energy barrier can be defined. The analogy between gradient banding and phase separation could be established in terms of the probability that events occur that initiate banding or phase separation. In case of banding, one would have to consider the probability distribution of waiting times to find a velocity profile with a sufficiently large amplitude and spatial extent to initiate banding.

The numerical solution of the above phenomenological equations of motion of stationary banded profiles in a wideangle cone-plate geometry, with the appropriate boundary conditions to account for the free fluid-air interface, have not been considered yet. Such calculations might explain the complicated flow profile that has been found in Britton and Callaghan (1997a).

Phase diagrams, where transition lines relate to banding transitions (not to thermodynamically driven transitions), have been constructed in Olmsted (1999b), without solving equations of motion. Both gradient and vorticity banding under controlled stress and controlled shear-rate conditions are considered, including coupling to concentration.

\section{The microscopic origin of strong shear thinning}

The van der Waals loop in Fig. 2 requires a very strong shear-thinning behaviour, such that $\sigma(\dot{\gamma})=\eta(\dot{\gamma}) \dot{\gamma}$ decreases with increasing shear rate $\dot{\gamma}$. Such a strong shear thinning behaviour can have different microscopic origins, depending on the type of system under consideration.

In some cases, strong shear thinning can be connected to the vicinity of a phase-transition line. To be explicit, we will discuss this phenomenon in terms of the isotropicnematic phase transition for rod-like colloids. A sketch of an experimental non-equilibrium phase diagram in the shear-rate versus concentration plane of rod-like colloids is given in Fig. 5a. The binodal marks the concentrations of coexisting paranematic and sheared nematic states (a "paranematic state" is a sheared isotropic state, that is, a state that would be isotropic in the absence of flow). The spinodal at higher concentration marks the points where the paranematic state becomes unstable against the nematic state on increasing the concentration. At the lower spinodal line, the nematic state becomes unstable against the paranematic state upon decrease of the concentration. The binodal and spinodals refer here to the thermodynamically driven isotropic-nematic phase transition, as modified by shear flow, and not to the earlier defined binodal and spinodal points related to gradient banding. On the spinodals, the collective rotational diffusion coefficient is zero and changes sign (Tao et al. 2006). Rotational diffusion is, therefore, very slow close to these spinodals. The circle in Fig. 5a marks the region where critical slowing 
Fig. 5 a The non-equilibrium phase diagram in the shear-rate versus concentration plane for a suspension of rod-like colloidal particles. The two phases here are a paranematic phase (a sheared isotropic phase) and a nematic phase. b A sketch of the shear-rate dependence of the shear stress for the two concentrations indicated by $A$ and $B$ in the phase diagram in a
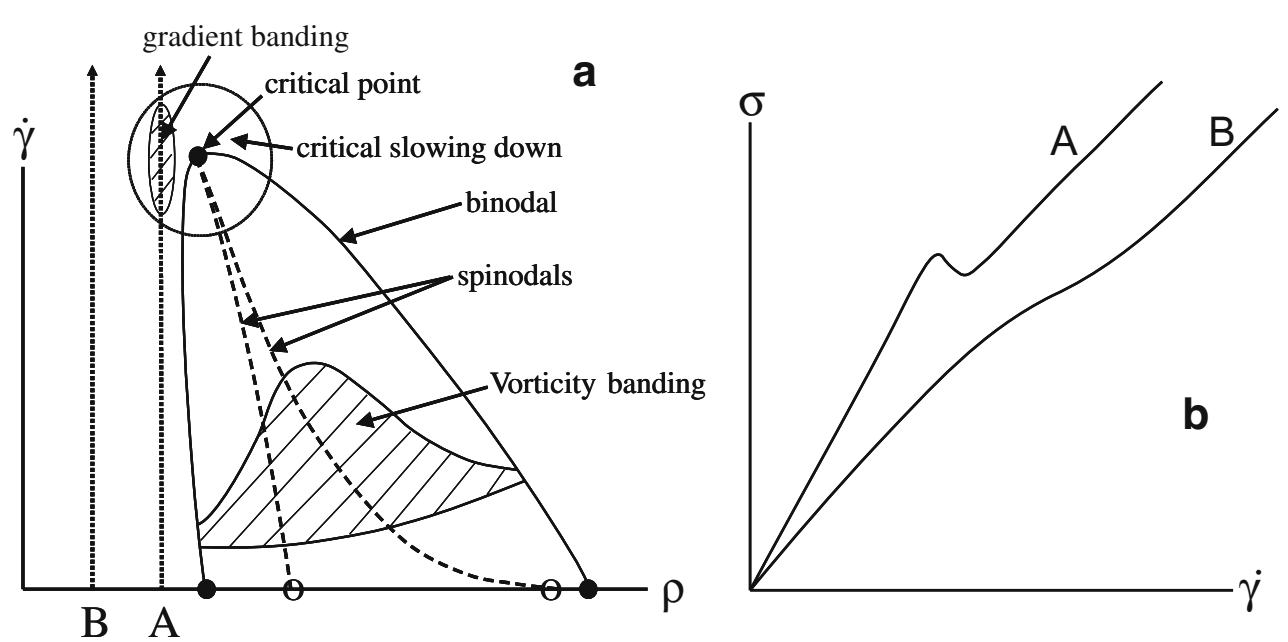

down of rotational diffusion is effective. On increasing the shear rate at the concentration "A" in Fig. 5a, such that the influence of critical slowing down on passing the critical point is effective, rotational Brownian motion no longer counter balances shear alignment. Thus, a small increase of the shear rate when entering the circle leads to a considerable increase in alignment and, thus, in a considerable decrease of the shear viscosity, possibly leading to a van der Waals loop. Gradient banding is thus expected within the shaded region marked "gradient banding" in Fig. 5a. This is how thermodynamics (modified by shear flow) couples to hydrodynamics. Whether gradient banding can occur within the two-phase region is yet an open question. At lower concentrations, such as concentration "B" in Fig. 5a, critical slowing down is not effective so that shear thinning is not strong enough to give rise to a van der Waals loop in the stress, as sketched in Fig. 5b. Doi-Edwards type of calculations (with an accurate closure for higher order moments of the orientation) indicate that the van der Waals loop for rigid rods with hard-core interactions is not very pronounced, so that gradient banding in such systems is probably not significant (Dhont and Briels 2003a; Olmsted and Lu 1999b). The van der Waals loop corresponding to the concentration " $A$ " in Fig. 5a is sketched in Fig. 5b. Vorticity banding occurs within the lower shaded region in Fig. 5a, which will be discussed in "Vorticity banding."

Theory on the behaviour of rod-like molecules, which (at least in part) describe the phase behaviour under shear-flow conditions, can be found in Olmsted and Goldbart (1992) and Dhont and Briels (2006). In Lenstra and Dhont (2001) and Grizzuti and Maffetone (2003), the location of the isotropic-nematic phase transition lines of liquid crystalline suspensions of fd virus and hydroxypropylcellulose in water have been studied by means of birefringence and rheology, respectively.

Pronounced van der Waals loops are found experimentally in worm-like micellar systems. As has been shown in computer simulations (Briels et al. 2004), two entangled, linear worms under sufficient tension can merge to form a longer worm. The typical configurations that two entangled worms undergo during the formation of a single, longer worm are sketched in Fig. 6 (a are computer simulation images and $b$ is a schematic of the merging process). For sufficiently high shear rates, two chains merge at the entanglement point. Right after merging, the point where the four arms meet has such a high free energy that it
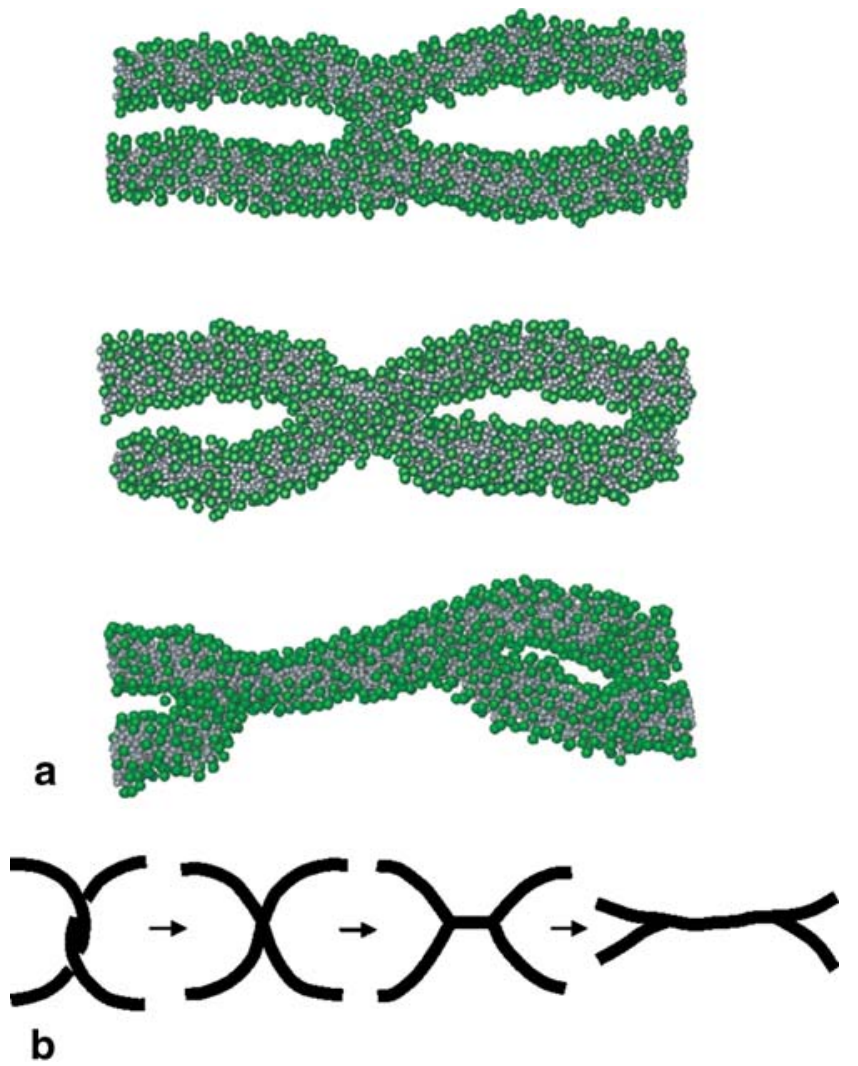

Fig. 6 a Computer images of merging worms (Briels et al. 2004), of which a schematic representation is given in $\mathbf{b}$ 
immediately splits into two $\mathrm{Y}$ junctions. In a later stage, the $\mathrm{Y}$ junctions move to the four end points to annihilate with two of the four high free-energy end caps. The resulting decrease of the number of entanglements gives rise to a decrease of the shear stress. In addition, the merging process might lead to a stronger alignment of the micelles along the flow direction, which also leads to a decrease of the shear stress with increasing shear rate, just as for rodlike colloids. If such an increase of alignment occurs, then the probability for merging of two end caps increases and leads to even more extended micelles. This may give rise to additional shear thinning. As the number of entanglements decreases with increasing shear rate, the above-described entanglement-merging process becomes ineffective at high shear rates. From then on the stress increases again with increasing shear rate. In addition, at higher shear rates the worms might be disrupted by shear forces leading to shorter worms. The length distribution of cylindrical micelles as a function of shear rate has not been studied experimentally. The experiments in Berret et al. (2001) and Münch et al. (1993) indicate that the worm length in solutions near the overlap concentration indeed increases with increasing shear rate. For the worm-like micellar system studied in Fischer and Callaghan (2001), there are indications that the microstructural order of the high shear-rate branch does not comply with a highly ordered nematic state. A possible explanation could be that worms are disrupted by strong flow or that tumbling of individual rod-like micelles leads to additional entanglements, resulting in a quasi-isotropic structure that supports a high stress.

The above discussed shear-thinning of worm-like micellar systems can be enhanced through the same mechanism as discussed for colloidal rods due to critical slowing down of orientational diffusion. The critical point can be connected to a shear-induced phase transition. In this case, discontinuous shear thinning can occur at sufficiently high shear rates. Examples of shear-induced transitions are the shear-induced isotropic-nematic transition in a thermotropic liquid-crystalline polymer (Mather et al. 1997) and worm-like micelles (Cappelaere et al. 1997; Schmitt et al. 1994). Apparently, such a discontinuous shear-thinning behaviour has not been connected to the occurrence of gradient banding.

Many of the above-described ingredients for shear thinning can be quantitatively accounted for by modifying the reptation model of entangled polymer dynamics to include breakage and recombination of polymer chains (Spenley et al. 1993; Cates et al. 1993). An overview of the existing theories and experiments on polymers and surfactant systems under flow is given in Olmsted (1999a), and the rheological behaviour of micellar systems, including banding transitions, is extensively discussed in Cates and Fielding (2006). The latter reference discusses several constitutive models to describe the sometimes complex banding phenomena in micellar systems, including temporal oscillations and chaos, as well as the role of structural memory effects.

Entanglements play an important role also in polymer systems, where, contrary to micellar systems, scission and recombination are absent. To the best of our knowledge, one of the first theories for polymers that explains a van der Waals type of behaviour of the stress as a function of shear rate was proposed in McLeish and Ball (1986). The strong shear-thinning behaviour is due to shear-induced disentanglement. No shear-curvature (or stress-diffusion) contribution has been considered here, so that stationary states required the investigation of the stability of infinitely sharp interfaces between the bands (McLeish 1987). A generalized Giesekus constitutive equation that incorporates nonaffine deformation of polymer chains predicts a severe shear-thinning behaviour leading to gradient banding (Vlassopoulos and Hatzikiriakos 1995). Here, the nonaffine deformation is due to "chain slip," leading to limited transmission of chain tension to its surroundings. The behaviour of polymer entanglements under flow conditions has very recently been described in Wang et al. (2007).

Entanglements in star-like polymers (or "resin particles"), consisting of a small hard core and a corona of polymer arms, lead to gradient shear banding as well. Recent computer simulations reveal the entanglement mechanism that underlies shear banding and the banding features of these systems (van den Noort and Briels). As the coronas encompass many degrees of freedom, which relax equally slow as the typical time scale on which the hard cores move, it is only feasible to perform simulations when an appropriate coarse graining can be devised. Important for the viscoelastic behaviour is the number of entanglements and their response to external stress. In a coarse-grained description, the number of entanglements $n_{i j}$ of two overlapping coronas of particles $i$ and $j$ characterizes the state of those coronas (van den Noort et al. 2007). The interparticle force $F_{i j}^{s t}$ due to overlapping coronas is taken equal to

$F_{i j}^{s t}=\alpha\left[n\left(r_{i j}\right)-n_{0}\left(r_{i j}\right)\right] \frac{d n_{0}\left(r_{i j}\right)}{d r_{i j}}$,

with $\alpha$ as a positive constant that characterizes the strength of entanglements and $n_{0}\left(r_{i j}\right)$ the number of entanglements of the two coronas when they are in equilibrium, given the distance $r_{i j}$ between the centers of the two particles. This choice has the advantage that the potential energy connected to the entanglements is given by a quadratic form $\frac{1}{2} \alpha\left[n\left(r_{i j}\right)-n_{0}\left(r_{i j}\right)\right]^{2}$, which leaves the distribution of particle positions unaffected. This makes it possible to tune thermodynamics and viscoelastic behaviour independently. 
The equation of motion for the number of stickers is simply taken equal to

$\frac{d n\left(r_{i j}\right)}{d t}=-\frac{n\left(r_{i j}\right)-n_{0}\left(r_{i j}\right)}{\tau}+F$,

where $F$ is a thermal force necessary to keep the temperature in the system constant. The relaxation time $\tau$ is reminiscent of the entanglement time in polymer networks. The two parameters $\alpha$ and $\tau$ in this model are supplemented by the radius $R$ of the core of the star polymers, for which an excluded interaction is assumed. The behaviour of two overlapping coronas is depicted in Fig. 7. Two coronas in equilibrium at short distance (Fig. 7a) are instantaneously displaced to a larger core-to-core separation (Fig. 7b). After the instantaneous displacement, the number of entanglements is the same as before the displacement. According to Eq. 15, this leads to an attractive entanglement force between the particles. Figure $7 \mathrm{c}$ and $\mathrm{d}$ shows the relaxation of the number of entanglements to the new equilibrium value according to Eq. 16, which is accompanied with a decrease of the entanglement forces between the particles. When two particles approach each other, there is a repulsive entanglement force. These time-dependent forces determine, to a large extent, the flow behaviour but essentially do not affect the thermodynamic properties of the system. The reason for strong shear thinning is that the coronas do not have time to develop entanglements when the shear rate is sufficiently large, such that $\dot{\gamma} \tau>1$. Such a shear-induced reduction of interaction forces leads to gradient banding. Typical banded flow profiles are shown in Fig. 8. As can be seen, the shear rates are independent of the applied, overall shear rate, as predicted by the minimal model discussed in "A minimal model: stress selection and banding kinetics." However, the concentration found in the two bands is different, as can be seen from Fig. 9. Despite the observed coupling to concentration, the stress selection rule and the lever rule seem to follow the predictions from the minimal model. Star polymers have experimentally been seen to shear-band just below the glass-/gel-transition points, where the shear rate within the low shear-rate band is nonzero (Holmes et al. 2004 and unpublished results of Dimitris Vlassopoulos and Jan Dhont, where bands have been found by means of heterodyne light scattering). It is very well feasible but still unexplored that, above the glass-/gel-transition point, one band is an elastic solid with zero shear rate, where the elastic stress balances the shear stress of the flowing band. The microstructural and viscoelastic properties of these star polymers and their glass/gel behaviour are described in Vlassopoulos et al. (1997), Stiakakis et al. (2002), and Vlassopoulos et al. (2001).

Strong shear thinning, giving rise to a van der Waals loop-like behaviour of the shear stress as a function of shear rate, has also been observed in transient networks where particles are dynamically connected to each other by telechelic polymers that act as bridging "stickers." The network consists of oil-in-water microemulsion droplets, bridged by hydrophobically end-modified water soluble polymers. The origin of the very strong shear-thinning behaviour of these networks is attributed to the shearinduced reduction of the residence time of the sticker polymer in a microemulsion droplet (Michel et al. 2001). The above-mentioned coarse model for star polymers could probably be applied also to gain in understanding on these transient networks. There are experimental indications, however, that fracture of these networks can occur at the wall of the shear cell (Molino et al. 2000).

A scenario that leads to gradient banding because of aggregation at a wall is described theoretically in Ajdari (1998). Here, the aggregated phase does not flow, and shear flow is assumed to peel off aggregated material at the
Fig. 7 a Two star polymers (or resin particles) at small core-tocore separation, where the polymer coronas are in equilibrium. b Overlapping coronas after an instantaneous displacement to a larger core-to-core separation. c and $\mathbf{d}$ The relaxation of the coronas to their new equilibrium state. Taken from van den Noort and Briels

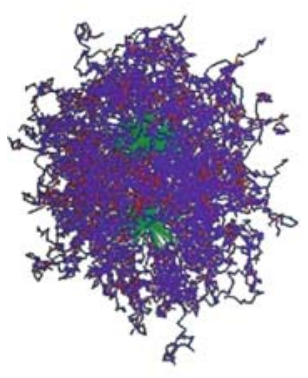

a

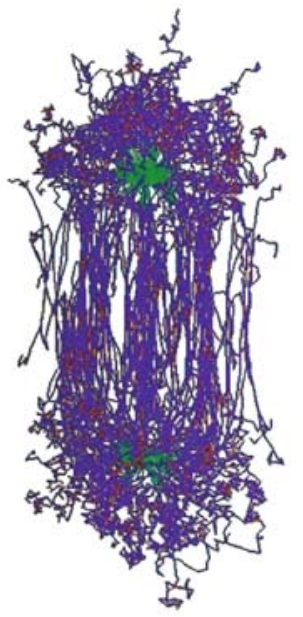

b

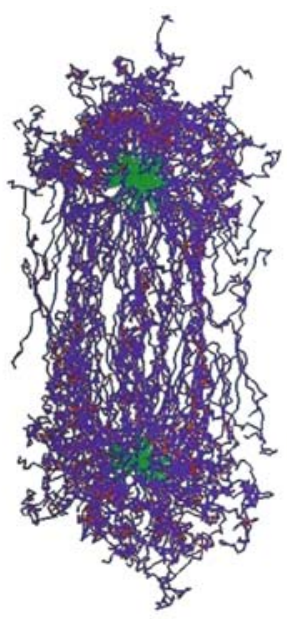

C

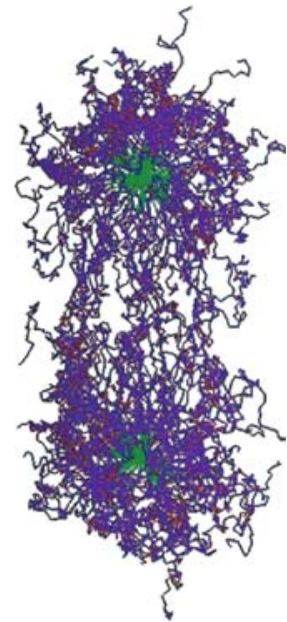

d 


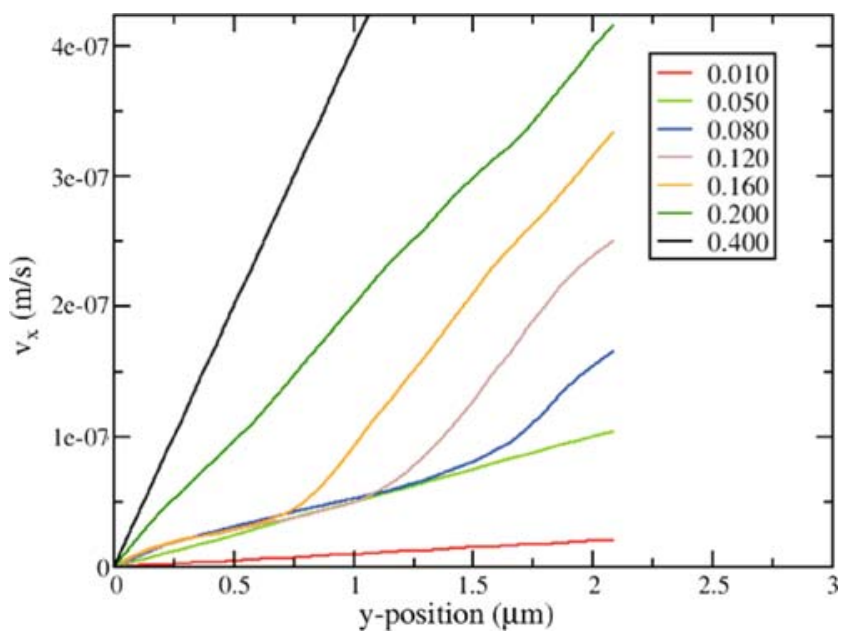

Fig. 8 Banded flow profiles found from coarse-grained simulations on star polymers for various overall shear rates as indicated in the inset in units of $\mathrm{s}^{-1}$. The range of the thermodynamic forces is about $100 \mathrm{~nm}$. Furthermore, $\alpha=5.1 k_{\mathrm{B}} T$ and $\tau=20 \mathrm{~s}$. Taken from van den Noort and Briels

aggregate-fluid interface. A one-dimensional model, with an empirical equation of motion for the interface location (that includes adsorption and peeling off), predicts stress jumps under controlled shear-rate conditions and shear-rate jumps under controlled stress conditions. It is not clear to which extent such a wall-induced aggregation mechanism plays a role in the experiments where banding is reported.

\section{Coupling to concentration}

The most serious omission in the minimal model discussed in "A minimal model: stress selection and banding kinetics" is the neglect of the concentration dependence of the shear viscosity (or any other degree of freedom that strongly

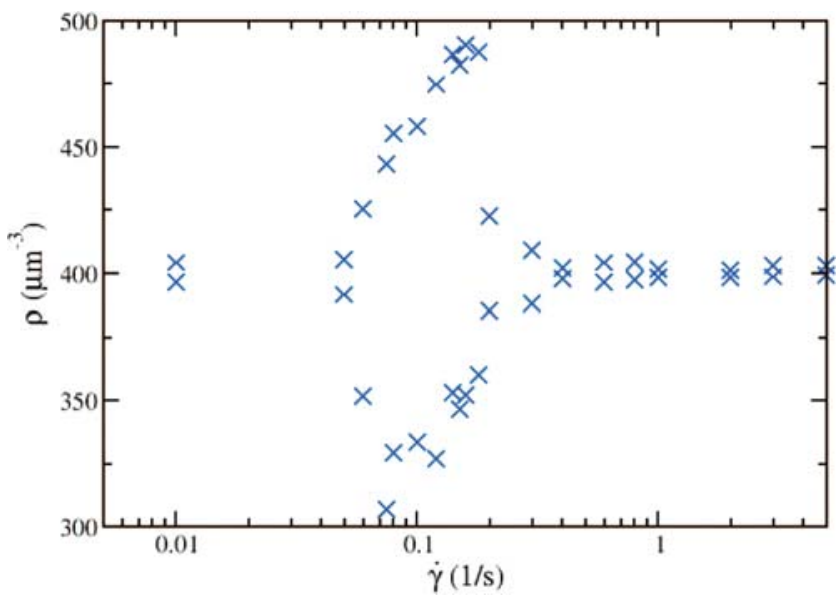

Fig. 9 The concentration of star polymers within the gap of the shear cell. Here, $\alpha=5.1 k_{\mathrm{B}} T$ and $\tau=20 \mathrm{~s}$. Taken from van den Noort and Briels couples to the stress, like orientational order in systems of an-isometric particles).

Such a "coupling to concentration" can have the consequence that gradient banding also occurs under controlled stress conditions. The possibility to vary the concentration within the two bands and the interface gives an extra degree of freedom for stress selection. Instead of the stress selection rule in Eq. 7, we now have, with $\rho(y)$ the local concentration

$$
\int_{y_{-}}^{y_{+}} d y \frac{\eta(\dot{\gamma}(y), \rho(y)) \dot{\gamma}(y)-\Sigma_{\text {stat }}}{\kappa(\dot{\gamma}(y))}=0,
$$

where, as before, $y_{+}$and $y_{-}$are positions within the high shear-rate and low shear-rate band, respectively. Due to the concentration entering the shear viscosity (and possibly the shear-curvature viscosity), it is not possible to rewrite this stress selection rule in terms of an integral with respect to shear rate as in Eq. 7 for the minimal model. Clearly, for each different concentration profile, there is a different stationary stress. Hence, the system can, in principle, adjust its concentration profile in such a way that the stationary stress $\Sigma_{\text {stat }}$ is equal to the applied stress in a stresscontrolled experiment. This explains the experimentally observed gradient banding under controlled stress conditions in Berret et al. (1998), Hu and Lips (2005), Eiser et al. (2000b), Lerouge et al. (2006), Cappelaere et al. (1997), and possibly in Roux et al. (1993). To fully describe the gradient-banding transition, including coupling to concentration and possibly orientational order, the Navier-Stokes equation must be complemented with appropriate diffusion equations. Banding of systems consisting of rod-like particles, including both coupling to concentration and orientational order, is discussed in Olmsted and $\mathrm{Lu}$ (1997, 1999b).

If concentration coupling is important, the stress of the stationary banded state is not strictly independent of the applied shear rate as for the minimal model. This can be easily understood as follows. Suppose that the profile with the lower applied shear rate in Fig. 3 solves the NavierStokes equation and the coupled mass-diffusion equation. If the concentration within the two bands differ, the flow profile for an elevated applied shear rate is now not obtained by a mere translation of the interface. As the total mass is conserved and the relative amount of the two bands changes, mass has to redistribute over the two bands. The concentration within the two bands is therefore depending on the applied shear rate. As the stress depends both on concentration and on shear rate, this affects the shear rates $\dot{\gamma}_{ \pm}$within the two bands. The shear rates in the bands are therefore depending on the applied shear rate, which renders the stationary stress $\Sigma_{\text {stat }}$ in the banded state also dependent on the applied shear rate(Olmsted 1999b; Olmsted and $\mathrm{Lu} \mathrm{1999b).} \mathrm{This} \mathrm{explains} \mathrm{the} \mathrm{sometimes}$ 
observed "tilted stress plateau" (Berret et al. 1998; Hu and Lips 2005; van der Gucht et al. 2006; Lerouge et al. 2000, 2006; Cappelaere et al. 1997; van den Noort and Briels 2007). Coupling to concentration has been discussed, within particulate models, in Olmsted and Lu (1999a, b) and Fielding and Olmsted (2003c). These models confirm that gradient banding is possible under controlled-stress conditions and predict a "positively sloped stress plateau." The situation is different when the stress couples to a nonconserved order parameter like orientational order, in which case no tilted stress plateau is expected.

A linear stability analysis of the coupled diffusion and Navier-Stokes equations has been performed by Schmitt et al. (1995). This analysis shows that coupling to concentration can lead to the initial formation of flow, banded in the gradient direction, even when $d \sigma\left(\dot{\gamma}_{0}\right) / d \dot{\gamma}_{0}$ is nonnegative. To analyze the effect of coupling to concentration on the initial instability of the flow, Fick's diffusion equation is first generalized to include driving forces for mass transport due to gradients in the shear rate. For small gradients in concentration, the mass flux $\mathbf{j}$ can be written as $\boldsymbol{j}=\rho \boldsymbol{u}-M \nabla \mu$, with $\rho$ the concentration, $\mathbf{u}$ the flow velocity, $\mu$ the chemical potential, and $M$ a positive transport coefficient. The chemical potential is a function of density and local shear rate: $\mu \equiv \mu(\dot{\gamma}, \rho)$. In principle, such a shear-rate-dependent chemical potential is ill defined, as shear flow is a nonconservative external field (it is explicitly shown by computer simulations in Butler and Harrowell 2002 that coexistence between crystals and fluid in flow cannot be described by means of a chemical potential). One possible way, however, to formally define a shear-rate-dependent chemical potential is to substitute shear-rate dependent order parameters into a known expression for the chemical potential. For example, in an expression for the chemical potential for rod-like colloids, one can simply substitute the shear-rate-dependent orientational order parameter, where its shear-rate dependence is obtained from independent equations of motion. It is not known how accurate such descriptions are. Assuming a shear-rate-dependent chemical potential, the mass flux is, thus, formally written as

$\boldsymbol{j}=\rho \boldsymbol{u}-M\left[\frac{\partial \mu(\dot{\gamma}, \rho)}{\partial \rho} \nabla \rho+\frac{\partial \mu(\dot{\gamma}, \rho)}{\partial \dot{\gamma}} \nabla \dot{\gamma}\right]$

As before, the fluid flow velocity is assumed to be in the $x$ direction. Only the magnitude of the velocity is allowed to vary, not its direction. Moreover, its magnitude is assumed to vary only in the gradient direction (the $y$ direction). Variations in the vorticity direction need not be considered, as the interest here is in instabilities extending along the gradient direction. Hence, the component $u$ of the velocity in the $x$ direction is written, as before, as $u(y, t)=\dot{\gamma}_{0} y+\delta u(y, t)$, where $\dot{\gamma}_{0}$ is the applied shear rate. Similarly, the density is assumed to vary only along the gradient direction: $\rho(y, t)=\rho_{0}+\delta \rho(y, t)$, with $\rho_{0}$ as the constant, initial concentration. Substitution of these expressions for the velocity and concentration into the expression for the flux and linearization with respect to $\delta u$ and $\delta \rho$ leads to the diffusion equation:

$$
\frac{\partial \delta \rho(y, t)}{\partial t}=D \frac{\partial^{2} \delta \rho(y, t)}{\partial y^{2}}+M \frac{\partial \mu\left(\dot{\gamma}_{0}, \rho_{0}\right)}{\partial \dot{\gamma}_{0}} \frac{\partial^{3} \delta u(y, t)}{\partial y^{3}}
$$

where it used the local shear rate equal to $\dot{\gamma}=\partial u(y, t) / \partial y$. Furthermore,

$D=M \frac{\partial \mu\left(\dot{\gamma}_{0}, \rho_{0}\right)}{\partial \rho_{0}}$

is Fick's diffusion coefficient. As the interest here is only in variations in the $y$ direction of the flow component $u$ in the $x$ direction, the only component of the stress tensor $\Sigma$ that contributes is $\sigma \equiv \Sigma_{y x}$ (apart from an irrelevant contribution $\left.\Sigma_{x x}\right)$. Here, we assume that the shear stress is given by the standard stress $\sigma$, which is now not just a function of the local shear rate $\dot{\gamma}=\partial u(y, t) / \partial y$, as in the minimal model, but also of the concentration. To leading order in $\delta \dot{\gamma}$ and $\delta \rho$, the deviation of the stress tensor from its initial value is thus given by

$\delta \sigma=\frac{\partial \sigma\left(\dot{\gamma}_{0}, \rho_{0}\right)}{\partial \dot{\gamma}_{0}} \delta \dot{\gamma}+\frac{\partial \sigma\left(\dot{\gamma}_{0}, \rho_{0}\right)}{\partial \rho_{0}} \delta \rho$

where the indices " 0 " refer to the homogeneously sheared system before banding occurred. The linearized NavierStokes equation thus reads (as before, $\rho_{m}$ is the mass density of the fluid):

$\rho_{m} \frac{\partial \delta u(y, t)}{\partial t}=\frac{\partial \sigma\left(\dot{\gamma}_{0}, \rho_{0}\right)}{\partial \dot{\gamma}_{0}} \frac{\partial^{2} \delta u(y, t)}{\partial y^{2}}+\frac{\partial \sigma\left(\dot{\gamma}_{0}, \rho_{0}\right)}{\partial \rho_{0}} \frac{\partial \delta \rho(y, t)}{\partial y}$

Equations 19 and 22 were derived in a somewhat more general setting in Schmitt et al. (1995). Here, we restrict ourselves to variations extending along the $y$ direction only. 
Disregarding the finite extent of the shear-cell gap width, the analysis for stability of variations along the gradient direction can be performed by substitution of

$\delta u(y, t)=\delta u_{0} \exp \{i k y-\Gamma t\}$,

$\delta \rho(y, t)=\delta \rho_{0} \exp \{i k y-\Gamma t\}$,

where $\delta u_{0}$ and $\delta \rho_{0}$ are the initial amplitudes of the variations of the velocity and concentration, respectively. Clearly, both the density and velocity are unstable when $\Gamma<0$. A finite gap width would require to write these variations as a sine/cosine-series with quantized wave vectors, as was done in the section on the minimal model. This is a technicality that would unnecessarily complicate the analysis in the present case. Substitution of Eq. 23 into Eqs. 19 and 22) gives

$$
\begin{aligned}
& \Gamma \delta \rho_{0}=D k^{2} \delta \rho_{0}+i k^{3} M \frac{\partial \mu\left(\dot{\gamma}_{0}, \rho_{0}\right)}{\partial \dot{\gamma}_{0}} \delta u_{0}, \\
& \rho_{m} \Gamma \delta u_{0}=k^{2} \frac{\partial \sigma\left(\dot{\gamma}_{0}, \rho_{0}\right)}{\partial \dot{\gamma}_{0}} \delta u_{0}-i k \frac{\partial \sigma\left(\dot{\gamma}_{0}, \rho_{0}\right)}{\partial \rho_{0}} \delta \rho_{0} .
\end{aligned}
$$

Eliminating $\delta \rho_{0}$ from the second equation gives a second order equation for $\Gamma$ :

$$
\begin{aligned}
\Gamma^{2}- & \Gamma \frac{k^{2}}{\rho_{m}}\left(\rho_{m} D+\frac{\partial \sigma\left(\dot{\gamma}_{0}, \rho_{0}\right)}{\partial \dot{\gamma}_{0}}\right)+ \\
& \frac{k^{4}}{\rho_{m}}\left(D \frac{\partial \sigma\left(\dot{\gamma}_{0}, \rho_{0}\right)}{\partial \dot{\gamma}_{0}}-M \frac{\partial \mu\left(\dot{\gamma}_{0}, \rho_{0}\right)}{\partial \dot{\gamma}_{0}} \frac{\partial \sigma\left(\dot{\gamma}_{0}, \rho_{0}\right)}{\partial \rho_{0}}\right)=0 .
\end{aligned}
$$

It follows from this result that $\Gamma$ is negative when (Schmitt et al. 1995)

$$
D \frac{\partial \sigma\left(\dot{\gamma}_{0}, \rho_{0}\right)}{\partial \dot{\gamma}_{0}}<M \frac{\partial \mu\left(\dot{\gamma}_{0}, \rho_{0}\right)}{\partial \dot{\gamma}_{0}} \frac{\partial \sigma\left(\dot{\gamma}_{0}, \rho_{0}\right)}{\partial \rho_{0}}
$$

When there is no coupling to concentration, that is, when $\partial \sigma\left(\dot{\gamma}_{0}, \rho_{0}\right) / \partial \rho_{0}=0$, this implies an instability either when (1) $D<0$ and $\partial \sigma\left(\dot{\gamma}_{0}, \rho_{0}\right) / \partial \dot{\gamma}_{0}>0$ or (2) $D>0$ and $\partial \sigma\left(\dot{\gamma}_{0}, \rho_{0}\right) / \partial \dot{\gamma}_{0}<0$. Case 1 corresponds to a spinodal instability leading to phase separation driven by thermodynamic forces, and case 2 corresponds to the mechanical gradient-banding instability that was found within the minimal model discussed before. When there is coupling to concentration, that is, when $\partial \sigma\left(\dot{\gamma}_{0}, \rho_{0}\right) / \partial \rho_{0} \neq 0$, there can be an instability, although $D$ and $\partial \sigma\left(\dot{\gamma}_{0}, \rho_{0}\right) / \partial \dot{\gamma}_{0}$ are positive. This is an instability that is purely driven by coupling with concentration and is absent within the minimal model. The intuitive explanation of the instability in this case is as follows. When $\partial \mu\left(\dot{\gamma}_{0}, \rho_{0}\right) / \partial \dot{\gamma}_{0}>0$, there will be mass transport in the direction of lower shear rates (see Eq. 18). Gradients in shear rate can be either due to fluctuations of the flow velocity or due to the shear-cell geometry. In a Couette cell, mass will be transported to the outer cylinder. When in addition $\partial \sigma\left(\dot{\gamma}_{0}, \rho_{0}\right) / \partial \rho_{0}>0$, the shear rate near the outer cylinder to which mass is transported will decrease, as the system will try to regain mechanical equilibrium where the shear stress is constant throughout the gap. Such a decrease in shear rate will lead to larger spatial gradients in the shear rate and thus enhances mass transport towards the outer cylinder. This self-amplifying mechanism leads to a high concentration and low shear-rate region near the outer cylinder and a low concentration and high shear-rate region near the inner cylinder. The self-amplifying mechanism is counter balanced by diffusion and a possible strong decrease of the stress on decreasing the shear rate. Diffusion will drive the system back to the homogeneous state and thus counter balances mass transport to regions with lower local shear rates. If the stress strongly decreases with decreasing shear rate, that is, when $\partial \sigma\left(\dot{\gamma}_{0}, \rho_{0}\right) / \partial \dot{\gamma}_{0}$ is positive and large, the decrease of the local shear rate in regaining mechanical equilibrium is small. This explains why the right-hand side in Eq. 26 (the driving force for the instability) should be larger than the left-hand side (the counter-balancing forces). When both derivatives on the right-hand side in Eq. 26 are negative, the same mechanism is at work, where now mass is transported to regions of higher shear rate.

It is not clear yet whether the above-mentioned selfamplifying mechanism, leading to an instability along the gradient direction for positive values of $D$ and $\partial \sigma\left(\dot{\gamma}_{0}, \rho_{0}\right) / \partial \dot{\gamma}_{0}$ indeed leads to a stationary gradientbanded state in the sense that regions (the "bands") exist within which the shear rate is essentially constant, independent of position. In any case, a stationary state is reached when gradients in concentration are sufficiently large so that Fickian diffusion counter balances the abovementioned driving force for mass transport connected to the shear-rate dependence of the chemical potential. In a gradient shear-banded state, such a balance is established within the interface. As the coupled diffusion equation and the Navier-Stokes equation constitute a nonlinear set of equations of motion, larger deviations from a homogeneously sheared state may stabilize the flow from further banding. To establish whether true shear-banded states are formed in the stationary state, the appropriate NavierStokes equation and diffusion equation should be solved numerically. To describe the stationary state, the NavierStokes equation must include the shear-curvature contribution to stabilize the system against the initial, rapid formation of large gradients in the flow velocity. When gradients in concentration are large, a similar higher order derivative in the concentration $\sim \partial^{4} \rho(y, t) / \partial y^{4}$ should be added to the diffusion equation. Such a higher order 
derivative was first introduced by Cahn and Hilliard in their analysis of the initial stages of spinodal decomposition (in which case $D$ is negative; Cahn and Hilliard 1958, 1959; see also Dhont 1996). The coefficient complying with this higher order derivative is commonly referred to as the Cahn-Hilliard square-gradient coefficient, which plays the same role as the shear-curvature viscosity in the sense that it stabilizes the system against rapid formation of very large spatial gradients. So far, no attempt has been made to analyze such a set of equations using a simple model for the concentration dependent shear stress and the shear-rate and concentration dependence of the chemical potential.

Other degrees of freedom that strongly couple to the shear viscosity can play a similar role as concentration. For example, for worm-like micelles and rod-like colloids, orientational order can be important (see "The microscopic origin of strong shear thinning"). Coupling to concentration and orientational order is discussed in Olmsted and $\mathrm{Lu}$ (1997, 1999b). Figure 1b in these references illustrates graphically the above-mentioned additional freedom that allows for gradient banding also under controlled stress conditions when coupling is important. Coupling of flow to the nonconserved composition of a binary mixture, where one of the components only exists under flow conditions, is discussed in Goveas and Olmsted (2001). Such a shearinduced new component that couples to the stress can lead to banding, depending on whether the stress or overall shear rate is controlled. In case of worm-like micelles, part of the high shear-rate branch, following the van der Waals loop, can be unstable due to coupling to the average worm length (Fielding and Olmsted 2004). A similar self-amplifying effect as for concentration coupling discussed before may be responsible for such an instability. As now the high shear-rate band is in itself unstable, there is no stationary gradient-banded state. This leads to time-dependent flow profiles under stationary applied shear rates (Fielding and Olmsted 2004).

\section{Gradient banding of shear-thickening systems}

Shear thickening can lead to a flow instability through a mechanism that is similar to that discussed in "Coupling to concentration." Suppose that the viscosity increases with increasing concentration. A local increase of concentration then leads to a local increase of the stress, which leads in turn to a decrease of the shear rate, as the system tends to restore mechanical stability. When migration occurs from regions of high shear rate to regions of low shear rate, this enhances the formation of an extending region of high concentration. Such a migration may be either due to (1) forces similar to those described in the previous subsection through the formal introduction of a shear-rate-dependent chemical potential or (2) by the hydrodynamically induced drift toward regions of lower shear rates for somewhat larger mesoscopic entities (see Frank et al. 2003 and references therein). The stationary state is now either a coexistence between a thick, high concentration phase and a thinner, low concentration phase, or the thick phase fills the entire volume, resulting in a homogeneously sheared state.

Discontinuous shear thickening can occur under controlled shear-rate conditions when a new, viscous phase is induced by the flow. This can be understood as follows. When the overall shear rate is fixed to a value above the critical shear rate where the new phase is induced, the formation of a more viscous phase in part of the system will reduce the corresponding local shear rate, so that the shear rate in the remaining low viscosity phase increases to a value even further above the critical shear rate. This leads to the additional formation of even more high viscous material. Due to this feed-back mechanism, under controlled shear-rate conditions, there will be a jump in the stress. Under controlled stress conditions, however, there is no discontinuity, as the new phase will form up to an extent that the overall system stress matches the applied stress. The shear-thickening systems studied in Herle et al. (2005), Porte et al. (1997), Berret et al. (2002), Boltenhage et al. (1997a, b), and Hu et al. (1998a, b) probably exhibit this phenomenon. Hysteresis can occur upon decreasing the shear rate below the critical shear rate, where the highviscous phase is meta-stable.

Some systems that exhibit a discontinuous shear-thickening transition exhibit vorticity banding. A possible mechanism that explains this, related to normal stresses in the gradient direction, will be discussed in the section on "Vorticity banding."

The jump in shear stress under controlled shear-rate conditions as a result of the feedback mechanism discussed above can become a continuous change when a Couette cell is used with a sufficiently large gap width. Upon increasing the overall shear rate, the natural variation of the shear rate within the gap leads to the formation of the viscous phase first close to the rotating inner cylinder, when the corresponding local shear rate exceeds the critical shear rate above which the new phase is formed. On further increasing the overall shear rate, the shear rate further away from the rotating inner cylinder will also exceed the critical shear rate, and more viscous phase is formed. In this way, continuous shear thickening would be observed in a shearrate interval that depends on the gap width of the cell, which should become narrower with decreasing gap width. This scenario is probably at the origin of what has been observed in Berret et al. (2000) for dilute surfactant solutions. The intrinsic variation of the local shear rate in a wide-gap Couette cell may also play a role in determining the flow profiles discussed in Coussot et al. (2002) for various glassy materials (bentonite suspensions, mayon- 
naise, silica suspensions, and white cement), where the Couette gap was $4 \mathrm{~cm}$ with an inner-cylinder radius of $8 \mathrm{~cm}$.

The structure of glasses and gels can be destroyed above the yield stress, which can lead to strong shear thinning. The flow curve would then have the form as sketched in Fig. 10a. Remember that the shear stress here is the stress of the homogeneously sheared system, where the shear rate is a constant throughout the system. In the banded state, one band is a non-flowing glassy or gel-like state that is elastically deformed to have the same shear stress as in the flowing band. Note that such elastic forces are not included in the minimal model discussed before. The assumption here is that no fracture or wall slip occurs. In high-density glassy systems, one can expect a strong coupling to concentration. A slight change of concentration of a liquid just below the glass transition results generally in a large change of the shear viscosity. As discussed in "Coupling to concentration" and at the beginning of the present subsection, such a coupling can lead to a flow instability along the gradient direction even in the absence of shear thinning. Coexistence of a nonflowing band with a flowing band is found in simulations on model glasses (Varnik et al. 2003), in experiments on pastes (Coussot et al. 2002) where the flow profile is probed by means of magnetic resonance imaging and in synthetic clay dispersions (Pignon et al. 1996) where the flow profile is visualized by means of color marking and video imaging. Yielding materials do not always shear thin when they start to flow. Examples of this are suspensions of the micro-gel particles studied in Cloitre et al. (2003).

Other mechanisms to explain (continuous or discontinuous) shear thickening observed for some worm-like micellar systems are proposed in Cates and Candau (2001). When the end-cap energy of cylindrical micelles is large, a dynamically linked network of micellar rings can be formed in the absence of shear flow. Applying a shear rate larger than the inverse of the slowest relaxation time of the network leads to a considerable distortion, giving rise to a strong increase of the stress. The strong increase of the stress at small shear rates observed experimentally requires feedback that could originate in changes of the statistics of scission-linking kinetics, as discussed in Cates and Candau (2001). The observed shear thickening of the worm-like micelles in Dehmoune et al. is attributed to the formation of stable Hstructured micelles (similar to the upper image in Fig. 6a), which are found from simulations in Briels et al. (2004).

The phenomenological theory proposed in Sollich et al. (1997, 1998), aiming to describe the rheology of jammed systems, is extended in Head et al. (2001) to describe, among other phenomena, the shear-induced "jamming transition" leading to the discontinuity in the flow curve as depicted in Fig. 10b. A somewhat speculative modecoupling approach predicts shear-induced glass formation below concentrations where a glass at rest is formed (Holmes et al. 2003). These theories do not include spatial variations of the flow velocity and therefore cannot describe banding transitions. For yielding systems, where the jamming transition is assumed to occur at rest and shear thins on flowing, as depicted in Fig. 10a, a phenomenological model is proposed in Picard et al. (2002). Here, a "fluidity parameter" is introduced, which interpolates between an elastic solid and a viscous fluid. Coupled equations of motion for the fluidity parameter and stress predict yield, gradient banding under controlled shear rate conditions, oscillations, and bottom and top jumps in shear rate under controlled-stress conditions, where the glassy phase does not flow except within a small layer near the walls.
Fig. 10 a The possible flow curve for a glassy or gel-like system with a yield stress. b Severe shear thinning of a shear-induced viscous phase. Here, $\dot{\gamma}_{c}$ is the "critical shear rate" above which the viscous phase is formed
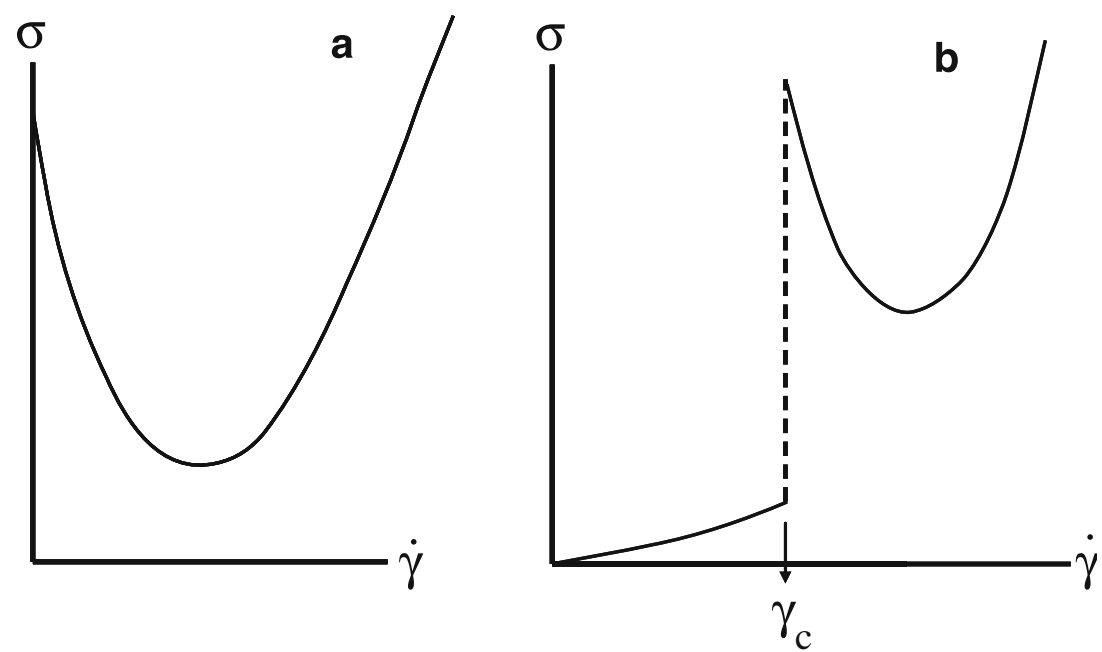


\section{Vorticity banding}

Vorticity banding has been observed in surfactant systems forming multi-lamellar vesicles (onions; Bonn et al. 1998; Wilkins and Olmsted 2006), crystallizing colloids (Chen et al. 1992), surfactant solutions (Fischer et al. 2002), dispersions of semirigid, rod-like colloids (Dhont et al. 2003; Kang et al. 2006), and nanotube suspensions where flow-induced, highly elastic clusters are formed (LinGibson et al. 2004). It should be mentioned that the solidlike clusters in Lin-Gibson et al. (2004) align along the vorticity direction in a log-rolling state. We will come back to this point later in this section. The large-scale bundles found in Vermant et al. (1999) in sheared sterically stabilized colloids indicate vorticity banding in these systems. The bands that are stacked along the vorticity direction (as sketched in the right Fig. 1) are visible either due to differences in optical birefringence (like for the rodlike colloids in Dhont et al. 2003 and Kang et al. 2006) and the onions in Wilkins and Olmsted (2006) or turbidity (like the worm-like micelles in Fischer et al. 2002). In some of these systems (Bonn et al. 1998; Chen et al. 1992), a van der Waals loop in the stress is found, so that it seems that both gradient and vorticity banding can occur.

It should be mentioned that band formation in this case is not related to a Taylor-Couette instability. The typical Taylor numbers in the above-mentioned experiments are much too small for Taylor banding to occur. In fact, Taylor banding has been detected for rod-like colloids (suspensions of fd-virus particles) for shear rates above $24 \mathrm{~s} \mathrm{~s}^{-1}$, which is much larger than the typical shear rates of $\left(\sim 0.2 \mathrm{~s}^{-1}\right)$ where vorticity banding is seen (private communication with Kyongok Kang). Taylor banding has also been observed in suspensions of rod-like boehmite colloids at shear rates of the order of $100 \mathrm{~s}^{-1}$ (Lenstra 2001).

Contrary to gradient banding, the mechanism that is at the origin of vorticity banding is less well understood. One approach to assess instabilities along the vorticity direction is to analyze an appropriate coupled diffusion equation and Navier-Stokes equation, similar to what has been done in "Coupling to concentration" for gradient banding. The additional stress tensor component that should now be taken into account is the vorticity-flow component $\sigma_{z x}$ of the stress tensor. The magnitude of the fluid flow velocity is now allowed to change along the vorticity direction as well, so that there is an additional shear rate $\dot{\gamma}_{z} \equiv \partial u(y, z, t) / \partial z$ in the vorticity direction. Omitting all derivatives with respect to $y$ in the two equations of motion, results in two possible instabilities along the vorticity direction. One instability occurs where $D<0$, in which case the density becomes unstable (Schmitt et al. 1995). This is just a spinodal instability. The other instability occurs where $\partial \sigma_{z x}\left(\dot{\gamma}_{0}, \dot{\gamma}_{z, 0} \rho_{0}\right) / \partial \dot{\gamma}_{z, 0},<0$, in which case the flow velocity becomes unstable. This instability is reminiscent of a gradient-banding instability along the vorticity direction, where, however, the initial shear rate $\dot{\gamma}_{z, 0}$ along the vorticity direction is arbitrarily small. It is as yet unclear whether such an instability can occur and whether it will lead to a stationary vorticity-banded state.

When in a stationary vorticity banded state the shear rate is essentially constant throughout the system, independent of position, the two types of bands will generally support different shear stresses. As the internal microstructural order is different for the two types of bands, they have different shear stresses when subjected to the same shear rate. A necessary condition for the existence of a stationary vorticity banded state is then that the flow curve (of the homogeneously sheared system) is multi-valued (Olmsted 1999b; Olmsted and Lu 1999b; Goveas and Olmsted 2001), as sketched in Fig. 11. An example of a multi-valued flow curve is that of a suspension of rod-like colloids, where the upper branch is the flow curve for an otherwise isotropic phase and the lower branch is the flow curve for the nematic phase with the same concentration. A multi-valued flow curve, however, is not a sufficient condition for vorticity banding, as it cannot explain the instability that leads to the initial formation of vorticity bands.

A mechanism that might explain the origin of vorticity banding (at least for some systems) is related to normal stresses along the gradient direction (Kang et al. 2006), similar to the hoop stresses that are at the origin of the Weissenberg effect in polymer systems. The microscopic origin of the Weissenberg effect in polymer systems is as follows (Pakdel and McKinley 1996; Groisman and Steinberg 1998; similar elastic instabilities are discussed in Muller et al. 1989, Larson et al. 1990, Shaqfeh et al.

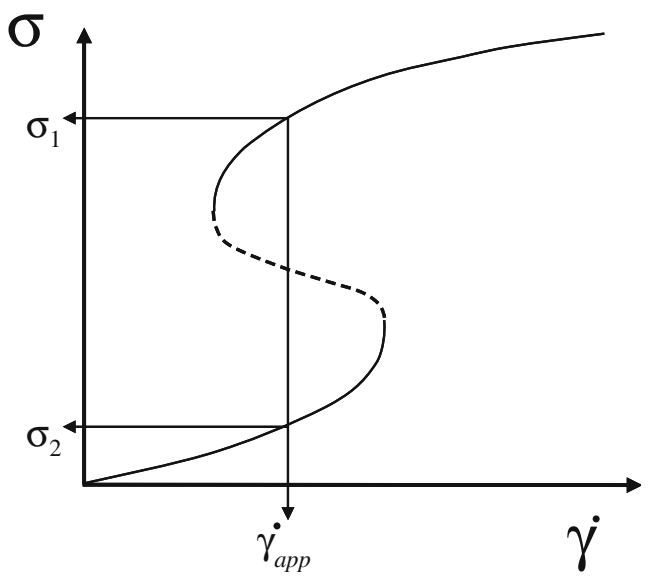

Fig. 11 A necessary condition for the existence of a stationary vorticitybanded state in case the shear rate is the same everywhere, independent of position, is that the shear-stress is multi-valued at the applied shear rate. In the stationary banded state, there are two types of microstructural order, corresponding to the two types of bands that can sustain different shear stresses $\sigma_{1}$ and $\sigma_{2}$ for the applied shear rate $\dot{\gamma}_{a p p}$ 
1992, and Shaqfeh 1996). Consider a polymer chain in a flow with gradients in the shear rate, like in the gap of a Couette cell as sketched in Fig. 12a, where the lines indicate equidistant flow velocities. In regions where the shear rate is relatively large, the polymer chain will be elastically stretched to a larger extent as compared to regions where the shear rate is small. This non-uniform elastic stretching of the chain is indicated by the dots on the polymer chain in Fig. 12a, which would be equidistant in the absence of flow. Averaging over many polymer chains, the non-uniform stretching of polymers gives rise to normal stresses directed toward the rotating inner cylinder. When these so-called hoop stresses are sufficiently large, they can give rise to a flow directed toward the inner cylinder. When the flowing fluid reaches the wall of the inner cylinder, the fluid will either turn up or down the wall. In the stationary state, this gives rise to bands that are in internal rolling motion, as sketched in Fig. 12b. This rolling flow is superimposed on the applied shear flow. Close to the meniscus, the flow toward the inner cylinder leads to an uprise of the meniscus, up to the inner cylinder, which is referred to as the "rod-climbing effect" (where "rod" refers to the inner cylinder). Such a rolling flow within the bands is also observed in case of vorticity banding in suspensions of rod-like colloids (solutions of fd-virus particles; Kang et al. 2006). The region where vorticity banding is found in these suspensions is indicated in Fig. 5 and lies entirely within the isotropic-nematic two-phase region. At a given concentration, vorticity banding is found above a "lower border shear rate" $\dot{\gamma}_{l}$ and below an "upper border shear rate" $\dot{\gamma}_{u}$, that is, banding occurs within a shear rate interval $\left(\dot{\gamma}_{l}, \dot{\gamma}_{u}\right)$. After a shear-rate quench into the vorticity-banding region, phase separation occurs, where inhomogeneities are formed. The idea is now that, instead of the polymer chains, the inhomogeneities that are formed during the initial stages of phase separation are nonuniformly stretched, as sketched in Fig. 12c. For the same reason as in the classic Weissenberg effect for polymers, the non-uniform elastic deformation of the inhomogeneities gives rise to hoop stresses that can be large enough to give rise to rolling flow. In addition, nonelastic normal forces could be produced by the inhomogeneities that contribute to the banding instability. Vorticity banding is then "the Weissenberg effect, where the role of polymer chains is played by inhomogeneities." That the mechanical properties of the inhomogeneities formed by initial phase separation in the rod-like colloidal system are at the origin of the banding instability is experimentally evidenced by (1) banding only occurs within the paranematic-nematic two-phase region, (2) banding kinetics is different whether inhomogeneities form an interconnected structure or whether the inhomogeneities are disconnected at the moment that banding sets in, and (3) banding ceases to occur at the top of the suspension as soon as the inhomogeneities disappear due to sedimentation (Dhont et al. 2003; Kang et al. 2006).

To minimize dissipation, a system subjected to simple shear flow will adapt its microstructural order along the gradient direction such that body forces $B_{\mathrm{y}}$ in that direction vanish (the index $y$ is used to indicate that these forces act along the gradient direction). In the present case, the internal structure of shallow inhomogeneities will be able to adjust to the flow such that $B_{\mathrm{y}}$ remains zero. When inhomogeneities become pronounced, however, such an adjustment of internal structure may not be possible anymore, resulting in a nonzero body force, leading to flow along the gradient direction. Consider a small increase of the amplitude of inhomogeneities. We ask for the amplitude of inhomogeneities under which such a change leads to flow in the gradient direction. Within the "Weissenberg scenario for vorticity banding," a nonzero
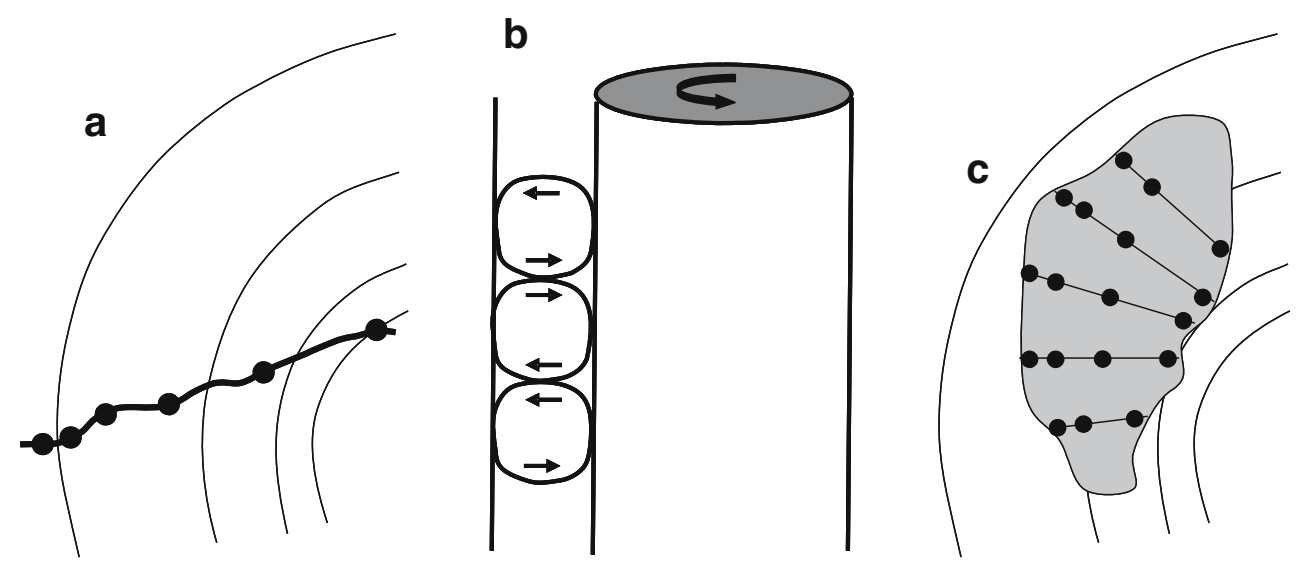

Fig. 12 a A polymer chain that is nonuniformly elastically stretched in a flow with gradients in shear rate. The lines indicate equidistant flow velocities in a Couette geometry. The dots on the polymer chain are used to indicate the nonuniform stretching. Without flow, these

dots would be equidistant. b Rolling flow within bands. c Nonuniform elastic stretching of inhomogeneities that can lead to the same rolling flow and accompanied vorticity-band formation as for the Weissenberg effect in polymer systems 
flow velocity $u_{y}$ along the $y$ direction (the gradient direction) initiates vorticity banding. As the discrete nature of the inhomogeneities along the flow direction is relatively unimportant, the gradient-component of the Navier-Stokes equation reads:

$\rho_{m}\left[\frac{\partial}{\partial t}+u_{y} \frac{\partial}{\partial y}+u_{z} \frac{\partial}{\partial z}\right] u_{y}(y, z, t)=B_{y}(y, z, t)$

where $u_{\mathrm{z}}$ is the flow velocity in the $z$ direction (the vorticity direction). Let $\delta u_{y}$ denote the small change of $u_{\mathrm{y}}$ due to a change of the amplitude of inhomogeneities and $\delta B_{y}$ the accompanied change of the body force. As $u_{\mathrm{z}}=0$ before banding occurs, linearization of the Navier-Stokes Eq. 27 leads to

$\rho_{m} \frac{\partial \delta u_{y}(y, z, t)}{\partial t}=\delta B_{y}(y, z, t)$

The $z$ dependence of both $\delta u_{y}$ and $\delta B_{y}$ is approximately sinusoidally $\sim \exp \{i k z\}$, where $\Lambda=2 \pi / k$ is the typical distance between inhomogeneities (as sketched in Fig. 13a). The $y$ dependence of $\delta u_{y}$ and $\delta B_{\mathrm{y}}$ is much more complicated and is related to the nonlinear deformation of the inhomogeneities along the gradient direction. Substitution of the forms

$$
\begin{aligned}
\delta u_{y}(y, z, t) & =\delta \bar{u}_{y}(y) \exp \{i k z-\Gamma t\}, \\
\delta B_{y}(y, z, t) & =\delta \bar{B}_{y}(y) \exp \{i k z-\Gamma t\} .
\end{aligned}
$$

into Eq. 28 leads to

$-\rho_{m} \Gamma \delta \bar{u}_{y}(y)=\delta \bar{B}_{y}(y)$.

Here, $\Gamma>0$ is the flow relaxation time in case no vorticity banding occurs, whereas $\Gamma<0$ is the initial "banding rate" when the system is unstable against vorticity banding.

A theoretical prediction for the behaviour of the body force $B_{\mathrm{y}}$ for rod-like colloids can be based on the equations of motion for the density and orientational order parameter and the constitutive equation for inhomogeneous suspensions as derived in Dhont and Briels (2002, 2003b). Here, the body force is expressed in terms of integrals over the probability density $\rho(\mathbf{r}, \mathbf{u}, t)$ for the position $\mathbf{r}$ of a rod and its orientation specified by the unit vector $\widehat{\mathbf{u}}$. The constitutive equation derived in Dhont and Briels (2003b) contains three distinct contributions: There is a Brownian body force, a contribution due to rod-rod interactions and a contribution due to direct coupling of flow to microstructural order. The Brownian body force and the coupling contribution are linear in the probability density, whereas the interaction contributions to the body force are bilinear. The probability density is now written as $\rho(\boldsymbol{r}, \widehat{\boldsymbol{u}}, t)=$ $A \widehat{\rho}_{0}(\boldsymbol{r}, \widehat{\boldsymbol{u}})+\delta A \widehat{\rho}_{1}(\boldsymbol{r}, \widehat{\boldsymbol{u}}, t)$, where $\widehat{\rho}_{0}$ and $\widehat{\rho}_{1}$ are renormalized densities, where their prefactors $A$ and $\delta A$ specify their amplitude. The density $\widehat{\rho}_{0}$ is supposed to lead to a zero body force in the gradient direction. We are looking for those amplitudes A, above which the probability density is not able anymore to adjust itself to the imposed shear rate/ stress such that the body force remains zero. Upon linearization of the above-mentioned contributions to the
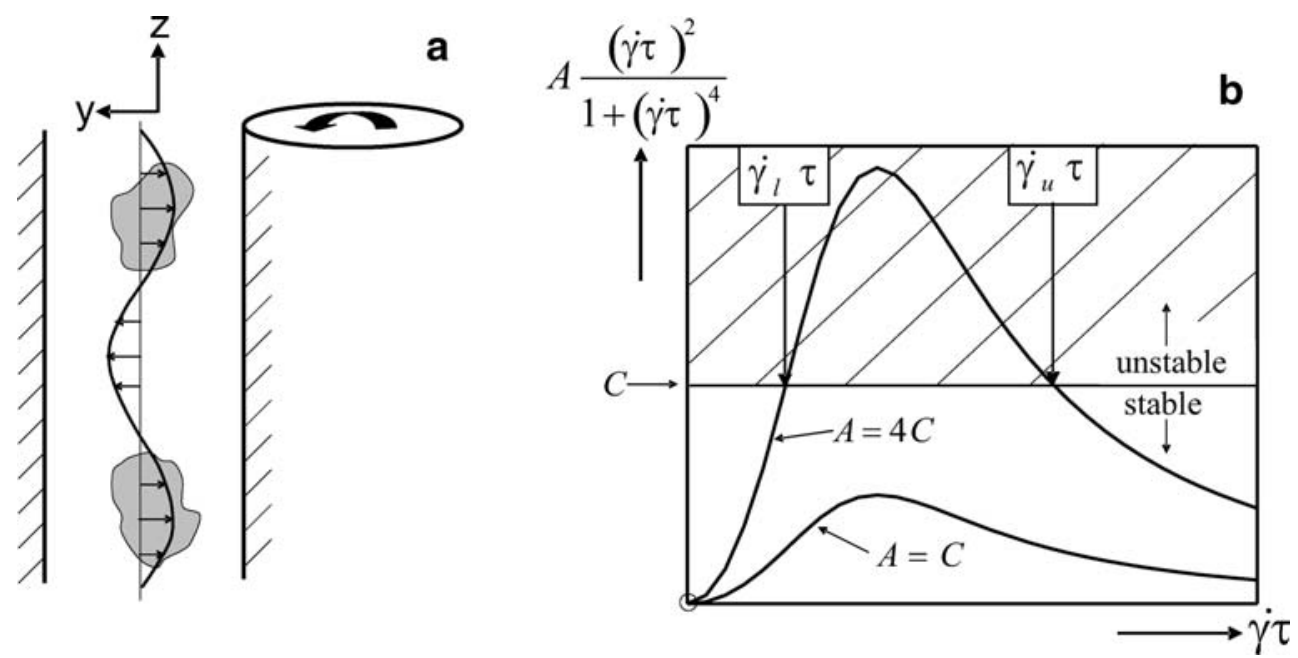

Fig. 13 a The flow field along the gradient direction in the initial stage of vorticity banding. The light gray inclusions are cross-sections of the inhomogeneities. The wave length $\mathrm{A}$ in the vorticity direction is of the order of a typical distance between inhomogeneities. b Stability diagram for vorticity banding within the "Weissenberg scenario." The quantity $A$ measures the magnitude of inhomogeneities. For the lower

curve, where $A=C<2 C$, the amplitude of inhomogeneities is not large enough to give rise to vorticity banding. For the upper curve, where $A=4 C>C$, the system is unstable in the shear-rate interval $\left(\dot{\gamma}_{l}, \dot{\gamma}_{u}\right)$, where $\dot{\gamma}_{l}$ is "the lower border shear rate" and $\dot{\gamma}_{u}$ is the "upper border shear rate." These shear rates correspond to the shear rates that bound the shaded region in the phase diagram in Fig. 5 
body force with respect to $\delta A$, the following possible contributions to $\delta B_{y}$ result. The linear terms in the density give rise to contributions that do not involve the amplitude $A$. Normal body forces vary like $\sim(\dot{\gamma} \tau)^{2}$ for small shear rates, where $\tau$ is the relaxation time of shear-induced deformations of inhomogeneities. For high shear rates, normal forces shear thin to a relatively small value. Hence, the linear terms in density give rise to contributions of the form $\sim(\dot{\gamma} \tau)^{2} f(\dot{\gamma} \tau) \delta A$, where $f$ tends to a nonzero constant for small shear rates and tends essentially to zero for high shear rates. For convenience, we shall use the simplest function with these limiting properties: $f(\dot{\gamma} \tau)=1 /$. $\left[1+(\dot{\gamma} \tau)^{4}\right]$. The rod-rod interaction contributions to the body force that are bilinear in the density similarly lead to contributions of the form $\sim(\dot{\gamma} \tau)^{4} f^{2}(\dot{\gamma} \tau) A \delta A$, as $\widehat{\rho}_{0}$ and $\widehat{\rho}_{1}$ are essentially equal. The linear change of the body force due to a slight change of the density is thus of the form

$\delta \bar{B}_{y}=\left\{C_{1}+C_{2} A \frac{(\dot{\gamma} \tau)^{2}}{1+(\dot{\gamma} \tau)^{4}}\right\} \frac{(\dot{\gamma} \tau)^{2}}{1+(\dot{\gamma} \tau)^{4}} \delta A$,

where the constant $C_{1}$ is linear in $\widehat{\rho}_{1}$ and $C_{2}$ is bilinear in $\widehat{\rho}_{0}$ and $\widehat{\rho}_{1}$, respectively. These constants are determined by the internal structural properties of the inhomogeneities. Without loss of generality, $A$ and $\delta A$ can be taken positive. Suppose that $\delta u_{y}$ is negative, implying that the flow is directed toward the inner cylinder at positions where inhomogeneities are present and a "backflow" occurs in between the inhomogeneities, as sketched in Fig. 13a. From Eqs. 30 and 31, the following instability criterion for which $\Gamma<0$ is then found:

$$
C_{1}+C_{2} A \frac{(\dot{\gamma} \tau)^{2}}{1+(\dot{\gamma} \tau)^{4}}<0
$$

As no banding is expected when interactions are absent (in which case $C_{2}=0$ ), the constant $C_{1}$ must be positive. Banding can thus occur when $C_{2}<0$. The instability condition can thus be rewritten as

$$
A \frac{(\dot{\gamma} \tau)^{2}}{1+(\dot{\gamma} \tau)^{4}}>C
$$

where $C=-C_{1} / C_{2}>0$. The corresponding stability diagram is given in Fig. 13b. The form $(\dot{\gamma} \tau)^{2} /\left[1+(\dot{\gamma} \tau)^{4}\right]$ has a single maximum at $\dot{\gamma} \tau=1$, with a maximum value of $1 / 2$. Hence, when $A<2 C$, there is no instability (see the lower curve in Fig. 13b, for which $A=C$ ). In this case, inhomogeneities are not sufficiently pronounced to produce body forces that lead to flow along the gradient direction.
That a minimum amplitude of inhomogeneities is required for vorticity banding is evidenced in the experiments on fd virus mentioned before (Kang et al. 2006) by the fact that after a shear-rate quench, banding is seen after some time, when phase separation has taken place long enough to produce sufficiently pronounced inhomogeneities. When $A>2 C$, an instability occurs only in a limited shear-rate range (see the upper curve in Fig. 13b, for which $A=4 C$ ). This is also seen in the experiments on fd virus (Kang et al. 2006). In fact, the lower and upper border shear rates $\dot{\gamma}_{l}$ and $\dot{\gamma}_{u}$ in Fig. 13 correspond to the shear-rate range that spans the vorticity-banding region (the dashed region) in the phase diagram in Fig. 5 for a given fd-virus concentration. A minimum shear rate is needed to produce sufficiently large deformations of inhomogeneities, which are not able to adjust to render $B_{\mathrm{y}}$ equal to zero. At sufficiently high shear rates, the inhomogeneities are stretched, rendering their linear dimensions in the gradient direction small, which probably enables an adjustment again where $B_{\mathrm{y}}=0$. A more precise analysis based on the constitutive relation derived in Dhont and Briels (2002, 2003b) might be feasible.

Besides suspensions of rod-like colloids, inhomogeneities can be identified in other systems that exhibit vorticity banding.

Vorticity banding has been seen in nano-tube suspensions (Lin-Gibson et al. 2004), which are partly aggregated. These aggregates are the necessary inhomogeneities to induce the hoop stresses leading to banding. The normal stress measurements in Lin-Gibson et al. (2004) indeed indicate that such stresses play a role in vorticity banding for these systems. The banding that is observed in these nano-tube suspensions might, however, be different from that found in other systems. Here, the bands align in a logrolling state along the vorticity direction instead of the flow direction. The bands consist of aggregated nano-tubes and therefore have a solid-like character. When these aggregates have a significant size, they will tend to align along the vorticity direction rather than the flow direction to minimize their internal elastic deformation energy. In case the aggregates are aligned along the flow direction, the shearing motion of the surrounding fluid gives rise to a high elastic deformation energy of the aggregates. This elastic energy can be released when the bands align along the vorticity direction, in which case the aggregates are able to partly accommodate to the shearing motion of the fluid by rotation along the vorticity direction. Due to the flow that is induced through their rotation, the elongated aggregates will possibly tend to form a regularly spaced structure. It is therefore feasible that the "log-roll banding" seen in these nano-tube suspensions is of a quite different nature as compared to the Weissenberg scenario discussed above. 
Vorticity banding has been observed in Bonn et al. (1998) for worm-like micellar systems (this system exhibits probably both gradient and vorticity banding). Either the worm-like micelles serve here as the inhomogeneities or a shear-induced nematic phase is formed, leading to the same kind of inhomogeneities as for the fd-virus suspensions discussed above.

Note that the vorticity-banded states can only exist in the presence of the inhomogeneities. A true stationary vorticitybanded state thus requires that the inhomogeneous system is inherently stable. When inhomogeneities are due to ongoing phase separation, the banded state disappears once the inhomogeneities begin to coalesce, and the system will ultimately attain its true stationary state. For the fd-virus suspensions in Kang et al. (2006), the true stationary state is therefore not the vorticity-banded state, but a state of coexistence of bulk volumes of a paranematic and a sheared nematic phase. Such a coarsening of inhomogeneities occurs after approximately a week, so that the vorticitybanded state in the fd suspensions are quite long-lived. In this case, the banded state could thus be referred to as a "quasi-stationary state."

Vorticity banding is some times observed when discontinuous (or very strong) shear thickening occurs (like for the worm-like micelles in Bonn et al. 1998, Wilkins and Olmsted 2006, and Fischer et al. 2002), where the shear stress exhibits a (quasi-)discontinuity, like in Fig. 10b (but not necessarily followed by shear thinning). Such a very strong shear-thickening behaviour can be due to the shearinduced formation of a new, viscous phase when the shear rate is increased above a critical shear rate (see the discussion in "Gradient banding of shear-thickening systems"). In Fischer et al. (2002), there are indeed clear indications for such a shear-induced new phase. Also, for crystalline colloids, there are indications of a shear-induced new phase that gives rise to vorticity banding (Chen et al. 1992). The formation of such shear-induced new phases may be accompanied by the formation of inhomogeneities. These inhomogeneities could then give rise to hoop stresses that lead to vorticity banding through the Weissenberg scenario as discussed above (personal communication with John Melrose).

A very recent proposition (Fielding 2007b) is that normal stresses giving rise to vorticity banding can be generated within the interface in a gradient-banded structure. In this case, first, a gradient-banded structure develops, which is then followed by the formation of vorticity bands. Vorticity banding is indeed sometimes found to occur in conjunction with a van der Waals loop in the stress, like for the multilamellar vesicles in Bonn et al. (1998) and the colloidal crystals in Chen et al. (1992). For the colloidal rods discussed above, however, no van der Waals-loop behaviour of the shear stress (just before vorticity banding occurs) has been found.

\section{Summary and conclusions}

A very simple model, referred to here as "the minimal model," explains a number of experimental observations in gradient-banding systems like:

- Gradient-shear banding occurs when the derivative of the shear stress of the homogeneously sheared system with respect to shear rate is sufficiently negative.

- Gradient banding occurs under controlled shear-rate conditions but (in a number of systems) not under controlled-stress conditions, where top and bottom jumps are seen.

- The shear rates within the bands are independent of the applied shear rate and a lever rule holds for the extent of the bands.

The essential, new ingredient in this model for the constitutive equation is a higher order spatial derivative of the flow velocity, which is characterized by the so-called shear-curvature viscosity. Alternatively, such nonlocal contributions can be introduced by adding a diffusive term to the equation of motion for the stress, which is characterized by a stress diffusion coefficient. These contributions to the stress stabilize the system against formation of very strong spatial gradients of the flow velocity.

Within the minimal model, the kinetics of band formation in the initial stage is predicted to exhibit similar characteristics as the Cahn-Hilliard scenario for gas-liquid spinodal decomposition. The initial banding kinetics can, however, be quite different from the "Cahn-Hilliard" scenario in case elastic contributions to the stress and/or coupling to concentration are important. In particular, there can be a broad wave-vector range where the growth rate is essentially constant. This implies that there is no welldefined wavelength that grows fastest during the initial stages of band formation.

The severe shear-thinning behaviour that is necessary for a decrease of the stress with increasing shear rate can be due to slow dynamics of a parameter that strongly couples to the stress. For rod-like colloids, this is the orientational order parameter that slows down near the nonequilibrium critical point in the shear-rate versus concentration phase diagram. For colloidal rods, however, the van der Waals loop is not very pronounced. The much more pronounced van der Waals loops found for micellar systems is possibly due to (1) the decreasing number of entanglements with increasing shear rate beyond a critical shear stress where entanglements merge and (2) the increase of the worm 
length with increasing shear rate. Transient entanglement forces can give rise to gradient banding of "resin particles", that is, small hard cores to which long polymer chains are attached. The concentration within the bands is different and strongly depends on the applied shear rate. Other mechanisms that lead to strong shear thinning for more particulate systems can be identified as well.

The most important omission in the minimal model is the concentration dependence of the shear viscosity. This concentration dependence in combination with mass diffusion allows for gradient banding also under controlledstress conditions, contrary to the predictions by the minimal model. A stability analysis that includes the concentration dependence of the shear stress and mass diffusion shows that there can be a new mechanism that leads to gradient banding that does not require strong shear thinning. The feedback mechanism and the conditions under which this instability occurs allow for a clear intuitive interpretation. A similar feedback mechanism can explain banding of strongly shear-thickening systems.

The origin of the vorticity-banding instability is less well understood as compared to gradient banding. When it is assumed that the shear rate is a constant throughout the shear cell, a necessary condition for banding is that the shear stress is a multivalued function of the shear rate. It is unclear, however, what mechanism is at the origin of vorticity banding in such cases. Rolling flow within the vorticity bands has been detected in suspensions of rod-like colloids, which hints to the importance of normal stresses in the gradient direction. On the basis of experiments on rodlike colloids, a mechanism is discussed, in which inhomogeneities are assumed to generate such normal stresses that give rise to vorticity banding. In a sense, this is the Weissenberg effect found for polymeric systems, where the role of the polymers is now played by inhomogeneities. This could also explain vorticity banding seen in systems that discontinuously (or very strongly) shear thicken due to the formation of a shear-induced viscous phase. The formation of the new phase involves the creation of inhomogeneities that in turn lead to normal stresses giving rise to vorticity banding. A primitive stability analysis is presented, based on an expression for the normal stress in inhomogeneous systems of rod-like colloids, that explains a few of the experimental observations in such systems. A very recent proposal is that normal stresses can be generated in the interface in a gradient-banded state. Here, the system will first form a gradient-banded state, after which vorticity bands develop from the interface, which are in internal rolling flow. More experimentation is needed to clarify the situation in particulate systems.

The shear-banding phenomena discussed in the present paper should be distinguished from the banding phenomena due to adiabatic heating. In materials that are subjected to very high shear rates, dissipation leads to temperature variations, which can be at the origin of band formation (for an overview see Grady (1994). In the present case, temperature variations are irrelevant due to the very low shear rates at which the shear-banding instabilities occur.

What has not been addressed in the present overview are time-dependent phenomena. This is a new and rapidly developing area of research that requires more experimental investigations to validate existing theoretical insight.

\section{References}

Ajdari A (1998) Rheological behavior of a solution of particles aggregating on the containing walls. Phys Rev E 58:6294-6298

Berret J-F (2005) Molecular gels. In: Weiss RG, Terech P (eds) Rheology of wormlike micelles: equilibrium properties and shear banding transition. Springer, Dordrecht, pp 235-275

Berret J-F, Porte G (1999) Metastable versus unstable transients at the onset of a shear-induced phase transition. Phys Rev E 60:42684271

Berret J-F, Porte G, Decruppe J-P (1997) Inhomogeneous shear flows of wormlike micelles: a master dynamic phase diagram. Phys Rev E 55:1668-1676

Berret J-F, Roux DC, Lindner P (1998) Structure and rheology of concentrated wormlike micelles at the shear-induced isotropic-tonematic transition. Eur Phys J B 5:67-77

Berret J-F, Gamez-Corrales R, Lerouge S, Decruppe J-P (2000) Shear thickening transition in surfactant solutions: new experimental features from rheology and flow birefringence. Eur Phys J E 2:343-350

Berret J-F, Gamez-Corrales R, Séréro Y, Molino F, Lindner P (2001) Shear-induced micellar growth in dilute surfactant solutions. Europhys Lett 54:605-611

Berret J-F, Lerouge S, Decruppe J-P (2002) Kinetics of shearthickening transitions observed in dilute surfactant solutions and investigated by flow birefringence. Langmuir 18:7279-7286

Boltenhage P, Hu YT, Matthys EF, Pine DJ (1997a) Observation of bulk phase separation and coexistence in a sheared micellar solution. Phys Rev Lett 79:2359-2362

Boltenhage P, Hu Y, Matthys EF, Pine DJ (1997b) Inhomogeneous structure formation and shear-thickening in worm-like micellar solutions. Europhys Lett 38:389-394

Bonn D, Meunier J, Greffier O, Al-Kahwaji A, Kellay H (1998) Bistability in non-Newtonian flow: rheology and lyotropic liquid crystals. Phys Rev E 58:2115-2118

Briels WJ, Mulder P, den Otter WK (2004) Simulations of elementary processes in entangled wormlike micelles under tension: a kinetic pathway to Y-junctions and shear induced structures. J Phys Condens Matter 16:S3965-S3974

Britton MM, Callaghan PT (1997a) Nuclear magnetic resonance visualization of anomalous flow in cone-and-plate rheometry. J Rheol 6:1365-1384

Britton MM, Callaghan PT (1997b) Two-phase shear band structures at uniform stress. Phys Rev Lett 78:4930-4933

Britton MM, Callaghan PT (1999) Shear banding instability in wormlike micellar solutions. Eur Phys J B7:237-249

Britton MM, Mair RW, Lambert RK, Callaghan PT (1999) Transition to shear banding in pipe and Couette flow of wormlike micellar solutions. J Rheol 43:897-909

Butler S, Harrowell P (2002) Factors determining crystal-liquid coexistence under shear. Nature 415:1008-1011 
Cahn JW, Hilliard JE (1958) Free energy of a nonuniform system. I. Interfacial free energy. J Chem Phys 28:258-267

Cahn JW, Hilliard JE (1959) Free energy of a nonuniform system. III. Nucleation in a two- component incompressible fluid. J Chem Phys 31:688-699

Callaghan PT (1999) Rheo-NMR: nuclear magnetic resonance and the rheology of complex fluids. Rep Prog Phys 62:599-670

Callaghan PT, Gil AM (2000) Rheo-NMR of semidilute polyacrylamide in water. Macro-molecules 33:4116-4124

Cappelaere E, Berret J-F, Decruppe J-P, Cressely R, Lindner P (1997) Rheology, birefringence and small-angle neutron scattering in a charged micellar system: evidence of a shear-induced phase transition. Pys Rev E 56:1869-1878

Cates ME, Candau SJ (2001) Ring-driven shear thickening in wormlike micelles? Europhys Lett 55:887-893

Cates ME, Fielding SM (2006) Rheology of giant micelles. Adv Phys 55:799-879

Cates ME, McLeish TCB, Marrucci G (1993) The rheology of entangled polymers at very high shear rates. Europhys Lett 21:451-456

Chen LB, Zukoski CF, Ackerson BJ, Hanley HJM, Straty GC, Barker J, Glinka CJ (1992) Structural changes and orientational order in a sheared colloidal suspension. Phys Rev Lett 69:688-691

Cloitre M, Borrega R, Monti F, Leibler L (2003) Glassy dynamics and flow properties of soft colloidal particles. Phys Rev Lett 90:068303-1-068303-4

Coussot P, Raynaud JS, Bertrand F, Moucheront P, Guilbaud JP, Huynh HT, Jarny S, Lesueur D (2002) Coexistence of liquid and solid phases in flowing soft-glassy materials. Phys Rev Lett 88:218301-1-218301-4

Decruppe JP, Cappelaere E, Cressely R (1997) Optical and rheological properties of a semi-dilute equimolar solution of cetyltrimethylammonium bromide and potassium bromide. J Phys II France 5:257-270

Decruppe J-P, Lerouge S, Berret J-F (2001) Insight in shear banding under transient flow. Phys Rev E 63:022501-1-022501-4

Dehmoune J, Decruppe J-P, Greffier O, Xu H (2007) Rheometric and rheo-optical investigation on the effect of the aliphatic chain length of the surfactant on the shear thickening of dilute wormlike micellar solutions. Rheol Acta 46:1121-1129

Dhont JKG (1996) Spinodal decomposition of colloids in the initial and intermediate stages. J Chem Phys 105:5112-5125

Dhont JKG (1999) A constitutive relation describing the shearbanding transition. Phys Rev E 60:4534-4544

Dhont JKG, Briels WJ (2002) Stresses in inhomogeneous suspensions. J Chem Phys 117:3992-3999

Dhont JKG, Briels WJ (2003a) Festschrift on the occasion of the 60th birthday of C.G. de Kruif, Viscoelasticity of suspensions of long, rigid rods. Coll Surf A Physicochem Eng Aspects 213:131-156

Dhont JKG, Briels WJ (2003b) Inhomogeneous suspensions of rigid rods in flow. J Chem Phys 118:1466-1478

Dhont JKG, Briels WJ (2006) Soft matter. In: Gompper G, Schick M (eds) Chapter 3: Rod-like Brownian particles in shear flow. Wiley-VCH, Weinheim, pp 147-283

Dhont JKG, Lettinga MP, Dogic Z, Lenstra TAJ, Wang H, Rathgeber S, Carletto P, Willner L, Frielinghaus H, Lindner P (2003) Shearbanding and microstructure of colloids in shear flow. Faraday Disscuss 123:157-172

Eiser E, Molino F, Porte G (2000a) Nonhomogeneous textures and banded flow in a soft cubic phase under shear. Pys Rev E 61:6759-6764

Eiser E, Molino F, Porte G, Pithon X (2000b) Flow in micellar cubic crystals. Rheol Acta 39:201-206

El-Kareh AW, Leal LG (1989) Existence of solutions for all Deborah numbers for a non-Newtonian model modified to include diffusion. J Non-Newtonian Fluid Mech 33:257-287
Fielding SM (2007a) Complex dynamics of shear banded flows. Soft Matter 3:1262-1279

Fielding SM (2007b) Vorticity structuring and velocity rolls triggered by gradient shear bands. Phys Rev E 76:016311-1-016311-8

Fielding SM, Olmsted PD (2003a) Early stage kinetics in a unified model of shear-induced demixing and mechanical shear banding instabilities. Phys Rev Lett 22:224501-1-224501-4

Fielding SM, Olmsted PD (2003b) Kinetics of the shear banding instability in startup flows. Phys Rev E 68:036313-036333

Fielding SM, Olmsted PD (2003c) Flow phase diagrams for concentration-coupled shear banding. Eur Phys J E 11:65-83

Fielding SM, Olmsted PD (2004) Spatiotemporal oscillations and rheochaos in a simple model of shear banding. Phys Rev Lett 92:084502-1-084502-4

Fischer E, Callaghan PT (2000) Is a birefringence band a shear band? Europhys Lett 50:803-809

Fischer E, Callaghan PT (2001) Shear banding and the isotropic-tonematic transition in wormlike micelles. Phys Rev E 64:0115011-011501-15

Fischer P, Wheeler EK, Fuller GG (2002) Shear-banding structure oriented in the vorticity direction observed for equimolar micellar solution. Rheol Acta 41:35-44

Frank M, Anderson D, Weeks ER, Morris JF (2003) Particle migration in pressure-driven flow of a Brownian suspension. J Fluid Mech 493:363-378

Georgiou GC, Vlassopoulos D (1998) On the stability of the simple shear flow of a Johnson-Segalman fluid. J Non-Newtonian Fluid Mech 75:77-97

Goddard JD (2003) Material instability in complex fluids. Ann Rev Fluid Mech 35:113-133

Goveas JL, Olmsted PD (2001) A minimal model for vorticity and gradient banding in complex fluids. Eur Phys J E 6:79-89

Grady DE (1994) Dissipation in adiabatic shear bands. Mech Mater 17:289-293

Grizzuti N, Maffetone PL (2003) Quiescent and flow-induced transitional behaviour of hydroxypropylcellulose solutions. J Chem Phys 118:5195-5200

Groisman A, Steinberg V (1998) Mechanism of elastic instability in Couette flow of polymer solutions: experiment. Phys Fluids 10:2451-2463

Head DA, Ajdari A, Cates ME (2001) Jamming, hysteresis, and oscillation in scalar models for shear thickening. Phys Rev E 64:061509-1-06509-16

Herle V, Fischer P, Windhab EJ (2005) Stress driven sgear bands and the effect of confinement on their structures - a rheological, flow visualization and rheo-SALS study. Langmuir 21:9051-9057

Holmes CB, Fuchs M, Cates ME (2003) Jamming transitions in a schematic model of suspension rheology. Europhys Lett 63:240

Holmes WM, Callaghan PT, Vlassopoulos D, Roovers J (2004) Shear banding phenomena in ultrasoft colloidal glasses. J Rheol 48:1085-1102

Hu YT, Lips A (2005) Kinetics and mechanism of shear banding in an entangled micellar solution. J Rheol 49:1001-1027

$\mathrm{Hu}$ YT, Boltenhagen P, Pine DJ (1998a) Shear thickening in lowconcentration solutions of wormlike micelles. I. Direct visualization of transient behavior and phase transitions. J Rheol 42:1185-1208

Hu YT, Boltenhagen P, Pine DJ (1998b) Shear thickening in lowconcentration solutions of wormlike micelles. II. Slip, fracture, and stability of the shear-induced phase. J Rheol 42:1209-1226

Hu H, Larson RG, Magda JJ (2002) Measurement of wall-slip-layer rheology in shear-thickening wormy micelle solutions. J Rheol 46:1001-1021

Imhof A, van Blaaderen A, Dhont JKG (1994) Shear melting of colloidal crystals of charged spheres studied with rheology and polarizing microscopy. Langmuir 10:3477-3484 
Kang K, Lettinga MP, Dogic Z, Dhont JKG (2006) Vorticity banding in rodlike virus suspensions. Phys Rev E 74:026307-1-026307-12

Kumar S, Larson RG (2000) Shear banding and secondary flow in viscoelastic fluids between a cone and plate. J Non-Newtonian Fluid Mech 95:295-314

Larson RG, Shaqfeh ESG, Muller SJ (1990) A purely elastic instability in Taylor-Couette flow. J Fluid Mech 218:573-600

Lenstra TAJ (2001) Dissertation: colloids near phase transition lines under shear. Utrecht University

Lenstra TAJ, Dhont JKG (2001) Shear-induced displacement of isotropic-nematic spinodals. J Chem Phys 114:10151-10162

Lerouge S, Decruppe JP, Humbert C (1998) Shear banding in a micellar solution under transient flow. Phys Rev Lett 81:5457-5460

Lerouge S, Decruppe J-P, Berret J-P (2000) Correlations between rheological and optical properties of a micellar solution under shear banding flow. Langmuir 16:6464-6474

Lerouge S, Argentina M, Decruppe JP (2006) Interface instability in shear-banding flows. Phys Rev Lett 96:088301-1-088301-4

Liberato MW, Nettesheim F, Wagner NJ (2006) Spatially resolved small-angle neutron scattering in the 1-2 plane: a study of shearinduced phase-separating wormlike micelles. Phys Rev E 73:020504-1-020504-4

Lin-Gibson S, Pathak JA, Grulke EA, Wang H, Hobbie EK (2004) Elastic flow instability in nanotube suspensions. Phys Rev Lett 92:048302-1-048302-4

Lu C-YD, Olmsted PD, Ball RC (2000) Effects of non-local stress on the determination of shear banding flow. Phys Rev Lett 84:642-645

Manneville S, Salmon J-B, Colin A (2004a) A spatio-temporal study of rheo-oscillations in a sheared lamellar phase using ultrasound. Eur Phys J E Soft Matter 13:197-212

Manneville S, Becu L, Colin A (2004b) High-frequency ultrasonic speckle velocimetry in sheared complex fluids. Eur Phys J AP 28:361-373

Manneville S, Colin A, Waton G, Schosseler F (2007) Wall slip, shear banding, and instability in the flow of a triblock copolymer micellar solution. Phys Rev E 75:061502-1-061502-11

Masselon C, Salmon J-P, Colin A (2008) Non-local effects in flows of wormlike micellar solutions. Phys Rev Lett (in press)

Mather PT, Romo-Uribe A, Han CD, Kim SS (1997) Rheo-optical evidence of a flow-induced isotropic-nematic transition in a thermotropic liquid-crystalline polymer. Macromolecules 30:7977-7989

McLeish TCB (1987) Stability of the interface between two dynamic phases in capillary flow in linear polymer melts. J Polym Sci 25:2253-2264

McLeish TCB, Ball RC (1986) A molecular approach to the spurt effect in polymer melt flow. J Polym Sci 24:1735-1745

Michel E, Appell J, Molino F, Kieffer J, Porte G (2001) Unstable flow and nonmonotonic flow curves of transient networks. J Rheol 45:1465-1477

Miller E, Rothstein JP (2007) Transient evolution of shear-banding wormlike micellar solutions. J Non-Newtonian Fluid Mech 143:22-37

Molino F, Appell J, Filali M, Michel E, Porte G, Mora S, Sunyer E (2000) A transient network of telechelic polymers and microspheres: structure and rheology. J Phys Condens Matter 12: A491-A497

Muller SJ, Larson RG, Shaqfeh ESG (1989) A purely elastic transition in Taylor-Couette flow. Rheol Acta 28:499-503

Münch Ch, Hoffmann H, Ibel K, Kalus J, Neubauer G, Schmelzer U, Selbach J (1993) Transient small-angle neutron scattering experiments on micellar solutions with a shear-induced structural transition. J Phys Chem 97:4514-4522

Olmsted PD (1999a) Dynamics and flow-induced separation in polymeric fluids. Curr Opin Colloid Interface Sci 4:95-100
Olmsted PD (1999b) Two-state shear diagrams for complex fluids in shear flow. Europhys Lett 48:339-345

Olmsted PD, Goldbart PM (1992) Isotropic-nematic transition in shear flow: state selection, coexistence, phase transitions, and critical behaviour. Phys Rev A 46:4966-4993

Olmsted PD, Lu C-YD (1997) Coexistence and phase separation in sheared complex fluids. Phys Rev E 56:R55-R58

Olmsted PD, Lu C-YD (1999a) Phase coexistence of complex fluids in shear flow. Faraday Discuss 112:183-194

Olmsted PD, Lu C-YD (1999b) Phase separation of rigid-rod suspensions in shear flow. Phys Rev E 60:4397-4415

Olmsted PD, Radulescu O, Lu C-YD (2000) Johnson-Segalman model with a diffusion term in cylindrical Couette flow. J Rheol 44:257-275

Pakdel P, McKinley GH (1996) Elastic instability and curved streamlines. Phys Rev Lett 77:2459-2462

Palberg T, Würth M (1996) Multiphase coexistence and non-linear rheology of colloidal dispersions as observed in an optical model capillary viscosimeter. J Phys I (France) 6:237-244

Picard G, Ajdari A, Bocquet L, Lequeux F (2002) A simple model for heterogeneous flows of yield stress fluids. Phys Rev E 66:051501-1-051501-12

Pignon F, Magnin A, Piau J-M (1996) Thixotropic colloidal suspensions and flow curves with minimum: identification of flow regimes and rheometric consequences. J Rheol 40:573-587

Porte G, Berret J-F, Harden JL (1997) Inhomogeneous flows of complex fluids: mechanical instability versus non-equilibrium phase transition. J Phys II France 7:459-472

Preis T, Biehl R, Palberg T (1998) Phase transitions in colloidal dispersions flowing through a cylindrical capillary. Prog Colloid Polym Sci 110:129-133

Pujolle-Robic C, Olmsted PD, Noirez L (2002) Transient and stationary flow behaviour of side chain liquid-crystalline polymers: evidence of a shear-induced isotropic-to-nematic phase transition. Eutophys Lett 59:364-369

Radulescu O, Olmsted PD, Lu C-YD (1999) Shear banding in reaction-diffusion models. Rheol Acta 38:606-613

Radulescu O, Olmsted PD, Decruppe JP, Lerouge S, Berret J-F, Porte G (2003) Time scales in shear banding of wormlike micelles. Europhys Lett 62:230-236

Ramos L, Molino F, Porte G (2000) Shear melting in lyotropic hexagonal phases. Langmuir 16:5846-5848

Rofe CJ, de Vargas L, Perez-González J, Lambert RK, Callaghan PT (1996) Nuclear magnetic resonance imaging of apparent slip effects in xanthan solutions. J Rheol 40:1115-1128

Roux D, Nallet F, Diat O (1993) Rheology of lyotropic lamellar phases. Europhys Lett 24:53-58

Salmon J-P, Colin A, Manneville S, Molino F (2003a) Velocity profiles in shear-banding worm-like micelles. Phys Rev Lett 90:228303-1-228303-4

Salmon J-B, Becu L, Manneville S, Colin A (2003b) Towards local rheology of emulsions under Couette flow using dynamic light scattering. Eur Phys J E 10:209-221

Salmon J-B, Manneville S, Colin A (2003c) Shear-banding in a lyotropic lamellar phase. Part 1: Time-averaged velocity profiles. Phys Rev E 68:051503

Salmon J-B, Manneville S, Colin A (2003d) Shear-banding in a lyotropic lamellar phase. Part 2: Temporal fluctuations. Phys Rev E 68:051504

Salmon J-B, Manneville S, Colin A, Pouligny B (2003e) An optical fiber based interferometer to measure velocity profiles in sheared complex fluids. Eur Phys J AP 22:143-154

Schmitt V, Lequeux F, Pousse A, Roux D (1994) Flow behaviour and shear induced transition near an isotropic/nematic transition in equilibrium polymers. Langmuir 10:955-961 
Schmitt V, Marques CM, Lequeux F (1995) Shear-induced phase separation of complex fluids: the role of flow-concentration coupling. Phys Rev E 52:4009-4015

Shaqfeh ESG (1996) Purely elastic instabilities in viscometric flows. Annu Rev Fluid Mech 28:129-185

Shaqfeh ESG, Muller SJ, Larson RG (1992) The effects of gap width and dilute solution properties on the viscoelastic Taylor-Couette instability. J Fluid Mech 235:285-317

Sollich P (1998) Rheological constitutive equation for a model of soft glassy materials. Phys Rev E 58:738-759

Sollich P, Lequeux F, Hébraud P, Cates ME (1997) Rheology of soft materials. Phys Rev Lett 78:2020-2023

Spenley NA, Cates ME, McLeish TCB (1993) Nonlinear rheology of wormlike micelles. Phys Rev Lett 71:939-942

Spenley NA, Yuan XF, Cates ME (1996) Nonmonotonic constitutive laws and the formation of shear-banded flows. J Phys II (France) 6:551-571

Stiakakis E, Vlassopoulos D, Loppinet B, Roovers J, Meier G (2002) Kinetic arrest of crowded soft spheres in solvents of varying quality. Phys Rev E 66:051804-1-051804-9

Tao Y-G, den Otter WK, Dhont JKG, Briels WJ (2006) Isotropicnematic spinodals of rigid, long thin rod-like colloids by eventdriven Brownian dynamics simulations. J Chem Phys 124:1349061-134906-10

Tapadia P, Ravindranath S, Wang S-Q (2006) Banding in entangled polymer fluids under oscillatory shearing. Phys Rev Lett 96:19600-1-19600-4

ten Brinke AJW, Bailey L, Lekkerkerker HNW, Maitland GC (2007) Rheology modification in mixed shape colloidal dispersions. Part I: Pure components. Soft Matter 3:1145-1162

van den Noort A, Briels WJ (2008) Coarse-grained simulations of elongational viscosities, super-position rheology and shear banding in model core-shell systems. Macromol Theory Simul $16: 742-754$

van den Noort A, Briels WJ (2007) Brownian dynamics simulations of concentration coupled shear banding. J Non-Newtonian Fluid Mech (in press) DOI 10.1016/j.jnnfm.2007.11.001

van den Noort A, den Otter WK, Briels WJ (2007) Coarse graining of slow variables in dynamics simulations of soft matter. Europhys Lett 80:28003-1-28003-5 van der Gucht J, Lemmers M, Knoben W, Besseling NAM, Lettinga MP (2006) Multiple shear-banding transitions in a supramolecular polymer solution. Phys Rev Lett 97:1083011-108301-4

Varnik F, Bocquet L, Barrat J-L, Berthier L (2003) Shear localization in a model glass. Phys Rev Lett 90:095702-1-095702-4

Vasquez PA, Cook LP, McKinley GH (2007) A network scission model for wormlike micellar solutions I. Model formulation and viscometric flow predictions. J Non-Newtonian Fluid Mech 144:122-139

Vermant J (2003) Large-scale structures in sheared colloidal dispersions. Curr Opin Colloid Interface Sci 6:489-495

Vermant J, Raynaud L, Mewis J, Ernst B, Fuller GG (1999) Largescale bundle ordering in sterically stabilized latices. J Coll Int Sci 211:221-229

Vlassopoulos D, Hatzikiriakos (1995) A generalized Giesekus constitutive model with retarda- tion time and its association to the spurt effect. J Non-Newtonian Fluid Mech 57:119136

Vlassopoulos D, Pakula T, Fytas G (1997) Ordering and viscoelastic relaxation in multiarm star polymer melts. Europhys Lett 39:617622

Vlassopoulos D, Fytas G, Pakula T, Roovers J (2001) Multiarm star polymer dynamics. J Phys Condens Matter 13:R855R876

von Hünerbein S, Würth M, Palberg T (1996) Microscopic mechanisms of non-linear rheology of crystalline colloidal suspensions. Prog Colloid Polym Sci 100:241-245

Wang S-Q, Ravindranath S, Wang Y, Boukany P (2007) New theoretical considerations in polymer rheology: elastic breakdown of chain entanglement network. J Chem Phys 127:0649031-064903-14

Wilkins GMH, Olmsted PD (2006) Vorticity banding during the lamellar-to-onion transition in a lyotropic surfactant solution in shear flow. Eur Phys J E 21:133-143

Yesilata B, Clasen C, McKinley GH (2006) Nonlinear shear and extensional flow dynamics of wormlike surfactant solutions. J Non-Newtonian Fluid Mech 133:73-90

Yuan X-F (1999) Dynamics of a mechanical interface in shear-banded flow. Europhys Lett 46:542-548 